\title{
Congress of Neurological Surgeons Systematic Review and Evidence-based Guidelines Update on the Role of Neuropathology in the Management of Progressive Glioblastoma in Adults
}

Abigail Goodman ( $\sim$ abigail.goodman@atriumhealth.org )

Atrium Health https://orcid.org/0000-0003-2596-5514

José E. Velázquez Vega

Children's Hospital of Atlanta

Chad Glenn

The University of Oklahoma

Jeffrey J. Olson

Emory University Hospital

\section{Research Article}

Keywords: Biomarker, EGFR, Immunohistochemistry, Infiltrating Glioma, MGMT Neuropathology, Molecular Testing, Progressive Glioblastoma, Recurrent Glioblastoma

Posted Date: January 7th, 2022

DOI: https://doi.org/10.21203/rs.3.rs-1028535/v1

License: (c) (1) This work is licensed under a Creative Commons Attribution 4.0 International License.

Read Full License 


\section{Abstract}

\section{Target population}

These recommendations apply to adult patients with progressive or recurrent glioblastoma (GBM).

\section{Question}

For adult patients with progressive glioblastoma does testing for Isocitrate Dehydrogenase (IDH) 1 or 2 mutations provide new additional management or prognostic information beyond that derived from the tumor at initial presentation?

\section{Recommendation}

Level III: Repeat IDH mutation testing is not necessary if the tumor is histologically similar to the primary tumor and the patient's clinical course is as expected.

Question For adult patients with progressive glioblastoma does repeat testing for MGMT promoter methylation provide new or additional management or prognostic information beyond that derived from the tumor at initial presentation and what methods of detection are optimal?

\section{Recommendation}

Level III: Repeat MGMT promoter methylation is not recommended.

Question For adult patients with progressive glioblastoma does EGFR amplification or mutation testing provide management or prognostic information beyond that provided by histologic analysis and if performed on previous tissue samples, does it need to be repeated?

\section{Recommendation}

Level III: In cases that are difficult to classify as glioblastoma on histologic features EGFR amplification testing may help in classification. If a previous EGFR amplification was detected, repeat testing is not necessary. Repeat EGFR amplification or mutational testing may be recommended in patients in which target therapy is being considered.

\section{Question}

For adult patients with progressive glioblastoma does whole genome or large panel sequencing provide management or prognostic information beyond that derived from histologic analysis?

\section{Recommendation}

Level III: Primary or repeat whole genome or large panel sequencing may be considered in patients who are eligible or interested in molecularly guided therapy or clinical trials. 


\section{Question}

For adult patients with progressive glioblastoma should immune checkpoint biomarker testing be performed to provide management and prognostic information beyond that obtained from histologic analysis?

\section{Recommendation}

Level III: The current evidence does not support making PD-L1 or mismatch repair (MMR) enzyme activity a component of standard testing.

\section{Question}

For adult patients with progressive glioblastoma are there meaningful biomarkers for bevacizumab responsiveness and does their assessment provide additional information for tumor management and prognosis beyond that learned by standard histologic analysis?

\section{Recommendation}

Level III: No established Bevacizumab biomarkers are currently available based upon the inclusion criteria of this guideline.

\section{Introduction}

\section{Rationale}

Glioblastoma is the most common primary brain tumor in adults, it is also one the most malignant and fatal brain cancers with a median survival time of only 15 months. $[1 ; 2]$ Because of the dismal prognosis, intensive standard therapy including surgical resection, radiotherapy, and chemotherapy is employed early in the course of the disease.[3] Despite this, nearly all glioblastomas will eventually recur and no effective standard treatment strategy against recurrent glioblastoma has been established.[4]

While highly variable among institution and clinical setting approximately $25-40 \%$ of patients with recurrent glioblastoma will undergo repeat surgery.[5-7] Unfortunately the radiation and adjuvant temozolomide therapy that is part of the current standard treatment for primary glioblastoma may produce tissue injury and necrosis that can be difficult to distinguish from recurrence radiologically.[810] These same therapy related effects that can cause radiographic uncertainty can also cause challenges for classification and grading of progressive glioblastoma histologically as well. Knowledge of the patient's clinical history and treatment status, neurosurgical impression, and the neuroradiologic findings are crucial.[8; 11] Additionally, it frequently means the patient has progressed after primary standard therapy and there may be increased interest in determining if the patient would be eligible for or may benefit from alternative treatment options. 
We evaluated the current literature addressing the diagnosis of progressive GBM, including histologic alterations present in response to therapy. Ancillary studies including immunohistochemistry and molecular diagnostic techniques in this setting will also be evaluated. With the success of immunotherapy and targeted treatment options in other tumor types there is a greater interest in more comprehensive molecular-genetic evaluation so literature pertaining to these subjects will be reviewed. Since the publication of the previous guideline the impact of select molecular features on the prognosis and progression of primary malignant brain tumors have been better recognized, some of which have been integrated into the revised 4th edition of the World Health Organization (WHO) Classification of Tumors of the Central Nervous System (CNS).[12]

\section{Objectives}

While the previously published guidelines[8] thoroughly delineated the histologic and immunohistochemical features of progressive glioblastoma limited studies were available at that time to assess what additional molecular diagnostics should be considered. Since that publication numerous studies looking at the role of ancillary and molecular studies for the diagnosis, prognosis, and treatment of glioblastoma have been published. This review addresses these advancements and reviews the recent literature to evaluate what ancillary testing is most appropriate in progressive glioblastoma to help guide treatment and prognosis and when ancillary testing is most appropriate.

\section{Methods}

\section{Writing Group and Question Establishment}

The evidence-based clinical practice guideline taskforce members and the Joint Tumor Section of the American Association of Neurological Surgeons (AANS) and the Congress of Neurological Surgeons (CNS) have prioritized an update of the guidelines for management of progressive glioblastoma. A series of writers were identified and screened for conflict of interest. This group in turn agreed on a set of questions addressing the role of neuropathology in the diagnosis of progressive GBM and conducted a systematic review of the literature relevant to the histopathologic diagnosis of progressive GBM in addition to immunohistochemical and molecular testing that can be used for either for diagnostic, prognostic, or treatment markers.

\section{Literature Search}

A search of PubMed, EMBASE, and Cochrane Library searches of the National Library of Medicine database of scientific literature published between July 1, 2012 and March 31, 2019. A broad search strategy using the following search terms was employed: "Progressive glioblastoma" OR "recurrent glioblastoma" OR "relapsing glioblastoma" OR "treated glioblastoma" OR "recurrent glioma" OR "relapsing glioma" OR "treated glioma" AND diagnosis OR pathology OR cytopathology OR "frozen section" OR radionecrosis OR "radiation necrosis" OR pseudoprogression OR gliosis OR immunohistochemistry OR proliferation OR genetics OR genomics OR prognosis OR accuracy OR "predictive value" OR sensitivity OR 
grading OR histology OR molecular OR genetic OR IDH OR biomarker OR ki-67 OR morphology." We limited our searches to human studies published in the English language. Key words were searched in multiple combinations. Links to "related articles" from highly relevant studies were utilized to broaden the search. Articles were also identified from the reference lists from articles uncovered in initial searches.

\section{Study Selection and Eligibility Criteria}

Original articles providing information to establish histopathologic diagnostic criteria for progressive glioblastomas and addressing immunohistochemical and molecular testing, and biomarkers in infiltrating gliomas and progressive glioblastomas were selected for review. A greater focus was put on studies looking at progressive and recurrent IDH-wildtype glioblastomas.

The citations were screened for the following inclusion and exclusion criteria:

\section{Inclusion Criteria}

Fully published peer-reviewed primary studies, that were published in English between July 1, 2012 and March 31, 2019 that focused on adult patients (>18 years of age).

\section{Exclusion Criteria:}

- Published in abstract form only

- In vitro studies only

- Animal studies only

- Studies focused on non-infiltrative gliomas or other CNS tumors

Those abstracts that met with the selection criteria mentioned above were retrieved in full text form. The adherence to the selection criteria were confirmed. The information was then used for construction of the evidence tables the text below.

\section{Data Collection Process}

The search resulted in 923 articles, which were reviewed yielding 283 potentially eligible articles. Links to "related articles" from highly relevant studies were utilized to broaden the search. Articles were also identified from the reference lists from references uncovered in initial searches. We also analyzed the references from prior evidence-based reports on glioblastomas and progressive glioblastomas.[8; 13] (See figure 1.)

\section{Assessment for Risk of Bias}

Inherent in research related to pathologic studies in patients with progressive glioblastoma is that they represent patients who were able to undergo a second surgery, likely those who were in a better state of health or younger patients at the time of 2 nd surgery. Additionally, these frequently represent patients with a relatively definable lesion, patients who present with more infiltrative disease are often less optimal 
patients for repeat surgery. The relatively recent identification of IDH mutations and MGMT promoter methylation status and their marked prognostic implications and the reclassification of infiltrating gliomas based on IDH and other molecular features has made it difficult to use historical samples and publications in which molecular testing was not performed. Thus there is a relatively limited time frame of data in which publications assessing progressive glioblastomas with molecular features incorporated into the results and makes using older studies that did not differentiate between these entities difficult to apply to the current classification system. Additionally, pathologic studies are very frequently retrospective, these biases are noted by the authors and the evidence level stratification does attempt to highlight these drawbacks to the reader.

\section{Classification of Evidence and Recommendation Levels}

The concept of linking evidence to recommendations has been further formalized by the American Medical Association (AMA) and many specialty societies, including the American Association of Neurological Surgeons (AANS), the Congress of Neurological Surgeons (CNS), and the American Academy of Neurology (AAN). This formalization involves the designation of specific relationships between the strength of evidence and the strength of recommendations to avoid ambiguity. We utilized the "Classification of Evidence on Diagnosis" to evaluate the literature and a summary of this classification can be viewed at https://www.cns.org/guidelines/guideline-development-methodology. Much of the pathology literature addressed below are well designed and studied large cohorts giving meaningful outcome data. However, due to the retrospective nature and lack of prospective validation will qualify them as Class III. Generally, Level I recommendations are based on Class I evidence, Level II recommendations are based on Class II evidence and Level III recommendations are based on Class III evidence.

\section{Results}

\section{Summary and Commentary on Previously Published Neuropathology Guideline}

As noted above this review is an update to the previously published guidelines for progressive glioblastoma by Brat et al.[8] It is useful to briefly review the questions and results from that paper.

The first topic addressed the diagnostic considerations in reporting progressive glioblastoma. A level III recommendation that the pathologist consider the patient's previous diagnosis and treatment, as well as the current clinical and neuroimaging features that led to a second biopsy or resection. In the setting of prior radiation and chemotherapy, it was recommended the pathologist adhere to strict histologic criteria for microvascular proliferation and necrosis in order to establish a diagnosis of a glioblastoma.

For patients undergoing biopsy or neurosurgical resection at the time of radiologic or clinical progression, reporting the presence and extent of progressive neoplasm as well as the presence and extent of necrosis 
within the pathologic material examined was recommended.[8] This recommendation continues to be supported in this update and with recent publications adding further credence to these as described below.

There is often a combination of radiation necrosis and progressive glioma in biopsy and resection specimens and it can be difficult to histologically assess disease status. Additional manuscripts published since the last version of this guideline refine the understanding of this issue. This was particularly emphasized by the study conducted by Holdhoff, et al., in which only "marginal agreement" by Fleiss' kappa statistics was observed when 48 pathologists ( $92 \%$ of whom were neuropathologists) reviewed 13 cases of suspected recurrence of glioblastoma. [14] Much of this may be due to differential understanding of the terminology used in this this study. The terminology of "active tumor", "inactive tumor", and "treatment effect" are not well established in the literature[14] yet this is commonly used terminology.

Multiple studies have aimed to determine what features give the greatest prognostic information on second resection specimen, methodology and results have varied. Azoulay et al., Dalle Ore et al., Hu et al., and Woodworth et al., show that the majority of re-resection specimens demonstrate a mixture of tumor and therapy related effects (ranging from $49-85 \%$ of specimens), with only a minority demonstrating absence of active residual tumor (ranging from $5-29 \%$ of patients). [9; $10 ; 15 ; 16]$ Azoulay et al. and Woodworth et al. demonstrated that patients in whom the re-resection specimen demonstrated only therapy related changes without active tumor had an increased survival.[15; 16] Hu et al. demonstrated a significant association between percent tumor and overall survival,[10] however Bagley et al. and Dalle Ore et al. were not able to reproduce these findings[5; 9] (See Table I). Another suggested marker of prognosis present in re-resection specimens has been the proliferative index as assessed by Ki-67 / MIB-1 immunohistochemistry. Okita et al. were able to demonstrate that MIB-1 indices significantly correlated with overall survival in a multivariate analysis $(p=0.004)[17]$ (See Table II). Thus, it is recommended that the percentage of viable tumor and the percentage of radiation necrosis is documented. If available the proliferative index as assessed by MIB-1 immunohistochemistry may also be informative, but it is not felt there is enough evidence at this time to include this as part of the recommendation (See Table II).

The second question in the prior version of this guideline addressed what ancillary studies are most useful in differentiating progression from treatment effect. Immunohistochemistry, including Ki-67, IDH, p53, and WT1, and genetic studies, specifically EGFR amplification or gain of chromosome 7, were selectively recommended for distinguishing neoplastic cells from atypical reactive cells in progressive glioblastoma.[8] This update does not alter this recommendation but numerous additional studies that have been published since then and have better elucidated the impact some of these features have on the behavior of infiltrating gliomas. Indeed, this was reflected in the World Health Organization 2016 Classification of Tumors of the Central Nervous System[12] which incorporates selected immunohistochemical and genetic features in the classification system. Thus, while the prior review focused on using these markers to determine the presence and in some cases quantity of tumor present, several of these studies are now indicated for classification and prognostic uses as well. 
The integration of ancillary studies is expected to expand with the publication of the next World Health Organization Classification of Tumors of the Central Nervous System with some of the expected updates already published in the form of CIMPACT-NOW update recommendations.[18] For the current review of progressive glioblastoma, criteria of the World Health Organization classification with the addition of the published cIMPACT-NOW update 3 recommendations will be used, since they represent a recent and updated international standard for classifying and grading.[12; 18] An additional cIMPACT-NOW update 5 was published after the designated time interval of the search for this guideline and the information in this document are not used to formulate the updated recommendations.[19]

It is of value to briefly expand on the evolution of the immunohistochemical and molecular markers alluded to in the second question of the prior guideline as it provides background on the new questions asked in this update as noted below. The 2016 WHO classification divides the diffuse gliomas into IDHwildtype astrocytomas, IDH-mutant astrocytomas, IDH-mutant and 1p/19q codelted oligodendrogliomas, and H3K27M-mutant diffuse midline glioma. A 3-tiered grading system is used in grading diffuse astrocytic gliomas, and a 2-tiered grading system of oligodendrogliomas.[12]

IDH-wildtype diffuse astrocytomas tend to arise in an older patient population with large majority of them presenting as de novo glioblastomas.[20] As such IDH-wildtype diffuse astrocytoma, WHO grade II and anaplastic astrocytoma (WHO grade III) are recognized as provisional entities in the $2016 \mathrm{WHO}$ and multiple studies have concluded that a substantial subset of these demonstrate an aggressive clinical course most akin to IDH-wildtype glioblastoma, WHO grade IV. Recently the cIMPACT-NOW update 3 addressed this by recommending that $I D H$-wildtype infiltrating astrocytomas that would histologically be classified as WHO grade II or III which carry and EGFR amplification, combined whole chromosome 7 gain and whole chromosome 10 loss, or a TERT promoter mutation be given an integrated diagnosis of "diffuse astrocytic glioma, IDH-wildtype, with molecular features of glioblastoma, WHO grade IV." It is critical to note that other IDH-wildtype glial tumors that may enter the differential have been reported to harbor TERT promoter mutations and thus histologic examination of the specimen remains critical.[18]

IDH mutant diffuse astrocytomas tend to arise in younger patient population and tend to present as either a diffuse astrocytoma WHO grade II or anaplastic astrocytoma WHO grade III. They are associated with TP53 mutations and ATRX alterations and are relatively slowly progressive.[12; 20; 21]

Diffuse midline glioma (H3K27-mutant) and H3 G34 mutant diffuse gliomas do not have IDH-mutations and occur predominantly in childhood and adolescents and are associated with an aggressive clinical course. Diffuse midline gliomas (H3K27M-mutant) are by definition WHO grade IV. While the 2016 World Health Organization Classification of Tumors of the Central Nervous System did not provide a separate classification or grade for H3 G34-mutant diffuse glioma, this mutation in a diffuse glioma indicates a high-grade biology with only modestly longer survivals than other IDH-wildtype glioblastomas. In most cases the assessment of prognostic genetic markers will have been performed on the initial specimen, however, if they were not performed previously they should be performed at the time of progression. Specific alterations that have current diagnostic, prognostic, or therapeutic implications in diffuse 
astrocytic gliomas include IDH1 and IDH2 mutations, EGFR amplification, whole chromosome 7 gain with concurrent whole chromosome 10 loss, TERT promoter mutation, CDKN2A homozygous deletion, 1p/19q co-deletion, and MGMT promoter methylation status. $[8 ; 12 ; 18]$

Question: For adult patients with progressive glioblastoma does testing for Isocitrate Dehydrogenase (IDH) 1 or 2 mutations provide new additional management or prognostic information beyond that derived from the tumor at initial presentation?

Study selection and Characteristics:

Four studies focused on IDH mutation status in primary and progressive glioblastoma were uncovered in the initial screen.[22; 23] Only two of these papers focused on the use of IDH mutations in recurrent glioblastoma. Two additional papers were identified from relevant review articles that addressed maintenance of IDH mutations during glioma progression[24; 25] (Table IV).

Results of individual studies, discussion of study limitations and risk of bias

Mutations in $I D H$ are frequent in lower grade astrocytomas as well as glioblastomas that progress from these lower grade precursor lesions. IDH mutations most commonly occur in $I D H 1$ as a substitution of histidine at R132, which accounts for approximately $90 \%$ of all IDH mutations.[12; 23] The IDH1 R132H mutation can be detected by either immunohistochemical or molecular methods. Approximately $10 \%$ of $I D H$ mutations are due to alternate mutations in IDH1 or on IDH2, including IDH1 R132C, IDH1 R132G, IDH1 R132S and IDH1 R132L and IDH2 R172K,[26] which can currently only be detected by molecular testing. IDH mutations tend to occur early in glioma development and they are retained upon recurrence, even after therapy. [24; 25; 27] IDH mutations contribute to glioma development through overproduction of the oncometabolite 2-hydroxyglutarate leading to induction of the HIF-1 pathway and genome wide histone and DNA methylation alterations.[12; 28-30] Testing for IDH1/2 mutations may be useful in the context of progressive glioblastoma when attempting to determine if residual disease is present in a resection specimen and to assess the approximate percentage of tumor.[8; 22] It is also feasible to use $I D H$ mutation testing to aid in frozen section diagnosis, however, the turnaround time is approximately 1 hour and may not be practical for all laboratories.[22] It should be noted that infiltrating gliomas, and glioblastomas are heterogeneous tumors and rare instances of alterations in IDH status from that seen in the original lesion have been noted, usually a loss of the IDH mutation[31-33](See Table III).

Synthesis

Due to the early occurrence and conserved nature of IDH mutations repeat testing is not necessary if the tumor is histologically similar to the primary tumor and the patient's clinical course is as expected.

Question: For adult patients with progressive glioblastoma does repeat testing for MGMT promoter methylation provide new or additional management or prognostic information beyond that derived from the tumor at initial presentation and what methods of detection are optimal? 
Study selection and Characteristics

Thirteen papers were uncovered in the screening process that discussed alterations in MGMT promoter methylation status in progressive/recurrent glioblastoma. Eleven were selected for inclusion as 1 was a review article and 1 paper only had four paired tumors and did not specifically note if MGMT promoter methylation status was retained in those four cases.

Results of individual studies, discussion of study limitations and risk of bias

Temozolomide, a chemotherapeutic agent that is part of the standard therapy for glioblastoma, and other alkylating agents cause DNA crosslinking through alkylation of the $0^{6}$ position guanine. These alkyl adducts are removed by $O^{6}$-methylguanine-DNA methyltransferase (MGMT). Methylation of the MGMT promoter is one of the major mechanisms for MGMT regulation and leads to transcriptional silencing. Thus, glioblastomas with MGMT promoter methylation and thus lower expression of MGMT would be expected to have better response to alkylating agents.[13; 34] MGMT promoter methylation has become a clinically relevant prognostic and predictive marker in patients with glioblastoma treated with temozolomide or other alkylating agents and is associated with a statistically significant improvement in progression free survival and overall survival in patients receiving standard therapy.[34; 35$]$

MGMT promoter methylation status is fairly consistent from original tumor presentation to recurrence, with no status change in approximately $66-82 \%$ of glioblastomas.[17; 36] No significant intra-tumor heterogeneity of MGMT promoter methylation status has been found.[36] Brandes et al. demonstrated that overall survival correlated with MGMT promoter methylation status determined at the primary surgery but not at recurrence and Okita et al. found no correlation between MGMT promoter methylation status at recurrence and survival time or progression free survival.[17; 36] A few earlier studies did show correlation with MGMT promoter methylation at recurrence and chemoresistance and survival,[37] however, the majority of the data suggests that repeat testing is not needed.

Multiple methods of $M G M T$ promoter methylation are available, including pyrosequencing, quantitative real-time methylation specific PCR, and methylation specific PCR. Recent studies have compared the multiple methods and tried to determine optimal stratification. The best performance was seen using a 3 tiered system of unmethylated, low level methylation, and high level methylation using either pyrosequencing or methylation specific PCR, with pyrosequencing being the preferred method.[34; 38-41]

An immunohistochemical stain for MGMT protein expression is also available, however MGMT protein expression can be upregulated by glucocorticoids, chemotherapy, and radiotherapy and thus my not reflect true MGMT status. It is also prone to high inter-observer variability and shows inconsistent correlation with clinical outcomes[38; 39; 42-44](See Table IV).

Synthesis 
Repeat MGMT promoter methylation testing does not need to be repeated upon recurrence. Either pyrosequencing or methylation specific PCR can be used to assess MGMT promoter methylation, with pyrosequencing being the preferred method. Immunohistochemical testing for MGMT protein expression is not recommended for clinical use.

\section{Question: For adult patients with progressive glioblastoma does EGFR amplification or mutation testing provide management or prognostic information beyond that provided by histologic analysis and if performed on previous tissue samples, does it need to be repeated?}

Study selection and Characteristics

Twenty articles were uncovered in the screening process of which 9 articles were selected for inclusion in this review and 1 additional article was identified from the references of the included articles. Reasons for exclusion from the included publications included animal or in-vitro studies, phase 1 clinical trials that were ended early or in which therapeutic outcomes were not discussed, review articles or single case studies, or did not address pathology (studies with only imaging data).

Results of individual studies, discussion of study limitations and risk of bias

EGFR is the most commonly amplified and overexpressed proto-oncogene in glioblastoma, with amplification present in approximately $40-50 \%$ of glioblastomas.[45-47] Amplification is primarily seen in de novo glioblastomas[45] and appears to be mutually exclusive of IDH mutations.[30; 48] EGFR is located on the short arm of chromosome 7 and encodes a cell-surface receptor tyrosine kinase. EGFR activation initiates signal transduction through several major pathways including RAS-MAPK and PI3KAKT signal transduction cascades leading to increased DNA transcription, anti-apoptosis, angiogenesis and cellular proliferation.[49-51] EGFR overexpression was found to contribute to gliomagenesis and poor survival in patients with glioblastoma.[52]

Mutations of EGFR are also seen in IDH-wildtype glioblastoma and appear to occur exclusively in the setting of EGFR amplified glioblastomas. EGFRvIII is the most common mutation in glioblastoma and results in the creation of a tumor-specific antigen that is detectable in $23-33 \%$ of IDH-wildtype glioblastomas,[53; 54] and in approximately $50 \%$ of EGFR amplified glioblastomas.[46; 55; 56] EGFRvIII is the result of a deletion of EGFR exons 2-7, which generates a constitutively active tyrosine kinase with a truncated extracellular domain.[46] The truncated extracellular domain creates a new unique targetable peptide sequence.[53; 57] EGFRVIV mutation is less common, seen in approximately $20 \%$ of EGFR amplified glioblastomas. EGFRvIV results due to deletion of the carboxyl terminal domain and also exhibits constitutive activation.[48]

Due to high frequency of EGFR alterations and the success in targeting EGFR in other tumors EGFR is an attractive target, however, EGFR inhibitors and an EGFRvIll vaccine have so far had disappointing results in glioblastomas.[58-60] It is reasonable to think that clinical trials targeting this pathway will continue and information regarding EGFR amplification and mutation may be desired. Felsberg et al., van den Bent 
et al., and Cioca et al. demonstrated that EGFR amplification was retained in recurrent glioblastomas after standard treatment and does not need to be retested. EGFRVIIImore commonly shows loss or reduced expression at recurrence and retesting should be considered if targeted therapy is being contemplated.[46; 52; 61] EGFR amplification can be detected by FISH, CGH, or PCR-based assays and EGFR mutations can be detected by PCR or IHC[8; 47; 61; 62] (See Table V).

Synthesis

Testing for EGFR amplification should be performed in cases of IDH-wildtype tumors that are difficult to grade as it may help classify the tumor as a glioblastoma. If a previous EGFR amplification was detected, retesting is not necessary. Repeat EGFR testing may be indicated for patients in which targeted therapy is being considered, particularly therapies targeting specific EGFR mutations.

\section{Question: For adult patients with progressive glioblastoma does whole genome or large panel sequencing provide management or prognostic information beyond that derived from histologic analysis?}

Study selection and Characteristics

16 studies were uncovered by the search criteria and ten studies were included in this review. The remaining studies were excluded because they were review articles (4) or were focused on lower grade infiltrating gliomas (2).

Results of individual studies, discussion of study limitations and risk of bias

Currently the only Federal Drug Administration (FDA) approved therapeutic agents for the treatment of progressive glioblastoma are bevacizumab, and in selected patients carmustine-wafers, thus patients are often encouraged to go on clinical trials.[63-65] Glioblastoma is molecularly heterogeneous and appears to be highly mutable with progressive glioblastoma displaying inherent or acquired resistance to treatment. $[63 ; 64 ; 66]$

Whole genome and large panel sequencing have expanded our understanding of the alterations that occur in de novo glioblastoma and progressive glioblastoma. Most studies demonstrate a gain of genetic alterations in recurrent glioblastomas[67] with approximately $17 \%$ of recurrent glioblastomas showing hypermutation[68]. Hypermutated tumors with alterations in the retinoblastoma (RB) and mammalian target of rapamycin pathways were noted after temozolomide therapy by Johnson et al.[24] while Wood et al. noted a subset of recurrent glioblastomas demonstrated an increased expression of CHI3L 1, TIMP1, and $C D 44$, whose expression has been associated with a more aggressive course[69]. Interestingly in one study $43 \%$ of recurrent gliomas showed a loss of $50 \%$ or more of the mutations present in the initial tumor, some of which were driver mutations, including TP53, ATRX, SMARCA4, and BRAF[24].

The increase in genetic understanding has also sparked hope that more effective novel or targeted therapies may be available for particular subsets of patients. To this end multiple studies looking at possible targets, alterations in recurrence, and clinical trials involving targeted therapies have been 
performed or are underway. Most studies were performed using next generation sequencing to asses genomic alterations either using data from whole transcriptome sequencing, whole exome sequencing, or targeted panels. Targeted panels have the advantage of being the most cost effective with the analysis of the data being the most straight forward and having relatively high specificity however the genes included on the targeted panels can vary and requires enrichment of the target regions. Whole exome sequencing requires enrichment of the exons and analysis of the data to ensure optimal processing and sequencing reaction. Whole genome sequencing while giving a comprehensive view of all alterations is the most expensive and requires the most data analysis and interpretation and many of the identified alterations may not be therapeutically targetable or relevant to diagnosis. Additional methods of genomic profiling that can be utilized include chromosomal microarray analysis and DNA methylation. It has become clear that progressive glioblastoma demonstrates an evolution of molecular alterations relative to primary glioblastoma.[24;67;69-71] Byron et al. demonstrated that genome wide molecular testing to guide therapy was feasible, and had promising results in 2 patients, in this study of a very limited size. [65] The ability of chosen therapies to cross the blood-brain barrier was taken into account in this study and is an important consideration when designing treatment recommendations. Most targeted therapies, particularly when used as single agent therapy, seem to have limited activity in the setting of progressive glioblastoma.[64] However, given the limited response to therapy in the recurrent setting repeat molecular testing would be of value in patients who are eligible for clinical trials based on a targeted therapy[65; 67; 68](See Table VI).

Synthesis

Primary or repeat whole genome or large panel sequencing should be considered in patients in whose management may be impacted including those who are eligible or interested in targeted therapy based on a particular oncogenic pathway anomaly or pathway member or for assessment of eligibility in clinical trials based on a particular molecular characteristic.

Question: For adult patients with progressive glioblastoma should immune checkpoint biomarker testing be performed to provide management and prognostic information beyond that obtained from histologic analysis?

Study selection and Characteristics

Twenty-seven articles were uncovered during the screening process, however the majority of these represented review articles and in vitro or animal models. Other studies were excluded due to a lack of pathology data (radiology studies), phase 1 studies that did not include pathology or outcomes, and studies focused on pediatric patients. Two additional studies were identified from references of review articles identified in the screening process. Ultimately eight studies were included in the review.

Results of individual studies, discussion of study limitations and risk of bias: 
Immune checkpoint inhibitors have shown marked success in the treatment of a variety of solid cancer types by blocking immune checkpoint signaling and allowing a T-cell response against the tumor.[72] Glioblastoma is known to cause host immunosuppression through a variety of mechanisms[63] and glioblastomas show frequent genetic and epigenetic alterations which potentially may produce numerous neoantigens,[72] thus immune checkpoint inhibitors sound like a promising treatment modality for progressive glioblastoma.

Variable PD-L1 staining patterns are seen in glioblastoma ranging from clear membranous staining to diffuse cytoplasmic staining[72; 73] and shows heterogeneity within the tumor.[72] The clinical significance of these differential staining patterns is yet to be elucidated but likely contributes to the wide range of PD-L1 expression in glioblastomas being reported, from 10-88\%.[73-76] A large proportion of tumors have been reported to demonstrate the diffuse pattern of staining, up to $88 \%$ of primary glioblastomas and $72 \%$ of recurrent glioblastomas .[73] While the clear membranous staining pattern is only seen in a subset of primary and progressive glioblastomas, approximately $37 \%$ of primary glioblastomas and $11-16.7 \%$ of progressive glioblastomas[73; 77], with Berghoff et al. noting a significant decrease in progressive glioblastomas demonstrating clear membranous PD-L1 expression. Heyneckes et al. found a significant decrease in both the PD-L1 mRNA expression and number of PD-L1 positive cells in progressive glioblastomas and that this reduction was more pronounced in patients who received extended temozolomide therapy. [72] The recent CheckMate 143 clinical trial, the first large randomized clinical trial of nivolumab, a PD-1 inhibitor, failed to extend overall survival in the setting of progressive GBM.[78-80] PD-L1 expression was not included as a criteria for inclusion in this study, and in the phase II CheckMate 143 studies while $68 \%$ of patients had PD-L1 expression over $1 \%$ only $27 \%$ of patients had PD-L1 expression greater than 10\%. Confounding these values is that the PD-L1 expression was measured on the primary resection, not the recurrent tumor.[78] A phase 1a study of atezolizumab in progressive glioblastoma also demonstrated dismal results with $100 \%$ of patients discontinuing therapy due to progressive disease. However, a more recent trial using pembrolizumab in patients with progressive glioblastoma as neoadjuvant therapy prior to re-resection and continuing as adjuvant therapy demonstrated improved overall survival (13.7 months) and progression free survival (3.3 months) when compared to patients receiving pembrolizumab as adjuvant therapy only after re-resection ( 7.5 months and 2.4 months respectively). Further studies will need to be done to further validate the use and efficacy of pembrolizumab in the neoadjuvant setting as this was a small study, it is also notable that PD-L1 expression was not a criteria and expression was not reported in either cohort.[81]

Another biomarker of interest is loss of mismatch repair (MMR) proteins and in 2017 the FDA approved pembrolizumab (Keytruda) for the treatment of unresectable or metastatic solid tumors harboring mismatch repair deficiency (dMMR) or microsatellite instability-high (MSI-H) regardless of site.[82] MMR enzymes, including MSH2, MSH6, MLH1, and PMS2, are involved in inducing programmed cell death in tumor cells damaged by alkylating agents, including temozolomide.[83] Multiple studies have shown that progressive glioblastoma has an increased prevalence of inactivating mutations of mismatch repair genes, particularly involving MSH6 and there is some evidence that MMR gene alterations are caused by or selected for by temozolomide therapy .[84-86] Indraccolo et al. found that the majority (78.5\%) of 
cases lacking MMR protein expression at recurrence had MGMT promoter methylation at diagnosis.[84] Loss of MMR enzyme expression is also associated with a hypermutant genotype, while this is a small subset of patients $(\sim 10 \%)$, it is hypothesized that the increased mutagenesis may make these tumors more immunogenic and thus more amenable to immunotherapy[74; 84] (See Table VII). However, the efficacy of pembrolizumab or other immune checkpoint inhibitors has yet to be investigated in MMR deficient progressive glioblastomas has yet to be fully investigated.

Synthesis

If immune checkpoint inhibitors are being considered PD-L1 expression or loss of MMR enzyme activity should be determined but due to the limited benefit demonstrated by immune checkpoint agents in glioblastoma (primary or progressive) standard testing is not currently necessary.

\section{Question: For adult patients with glioblastoma are Bevacizumab biomarkers available and should they be performed in the setting of progressive glioblastoma?}

Study selection and Characteristics

Numerous studies (greater than 75) looking at Bevacizumab were identified however most were clinical trial papers, without examination of pathology, studies looking at radiologic biomarkers, or review articles. Eight articles looking at tissue biomarkers were identified and included in this review.

Results of individual studies, discussion of study limitations and risk of bias

Glioblastoma demonstrates marked up-regulation of VEGF-A and displays rapid vascularization.[4; 87] Bevacizumab is a monoclonal antibody against vascular endothelial growth factor (VEGF) that has been approved for treatment of recurrent glioblastoma. However, bevacizumab has not been shown to improve OS and there is concern that glioblastomas treated with bevacizumab are more aggressive and show increased infiltration.[4; 88; 89] A subset of patients do show a favorable clinical response following bevacizumab and biomarkers are being investigated to identify these patients.[4] Choi et al. and Hovinga et al. both found that classical subtype glioblastomas did not respond as well to bevacizumab.[4; 47] Choi et al. and Erdem-Eraslan et al. identified possible biomarkers in predicting response to bevacizumab (COL4A2) and bevacizumab combined with lomustine (FMO4/OSBPL3), however, both of these are retrospective studies on primary tumor specimens and further studies need to be performed to confirm these findings.[4; 90] YKL-40 mRNA expression and plasma levels have been found to be associated with a worse response to carmustine plus bevacizumab or bevacizumab therapy alone, respectively[91; 92] in retrospective studies but no prospective studies have been performed. At this time no established biomarkers are available to predict response to bevacizumab[88; 93; 94] (See table VIII).

Synthesis

Bevacizumab biomarker testing at this time remains experimental, markers which may be indicative of response include YKL-40, COL4A2, and FMO4 expression although confirmatory studies are warranted. 


\section{Discussion}

If prior ancillary and molecular testing including $I D H$ mutation status, MGMT promoter methylation status, and other relevant testing including chromosomal alterations and histone mutations were not performed on the primary resection specimen, it is recommended they be performed at the time of progression for definite classification.

All of the recommendations presented within this review are based on class III data, usually due to the retrospective nature of the studies or small cohort size, however, the recommendations are based upon multiple class III studies whose results are coherent. Repeat testing of commonly assessed alterations, including IDH mutation, MGMT promoter methylation, and EGFR amplification, usually do not need to be repeated upon progression as they tend to remain stable, or in the case of MGMT promoter methylation alterations present at the time of progression are of unclear clinical and therapeutic significance. Less common studies including EGFR mutations, large panel sequencing, and immune checkpoint biomarker testing may be warranted in patients who are interested in targeted therapy, immunotherapy, or other select clinical trials where presence or absence of a particular molecular marker is included in the eligibility criteria. While a few studies have shown benefit from targeted therapy and immunotherapy there is currently not enough evidence to suggest that these studies be considered part of standard workup. This is particularly true in the case of immune checkpoint inhibitors, in which the majority of clinical trials have been disappointing and the relevance of PD-L1 or MMR expression in the efficacy of these agents is unclear as the clinical trials have not required PD-L1 expression or MMR loss as a criteria and no association between PD-L1 expression and response.[72; 73; 78; 80; 95-97] However, promising results have recently emerged using pembrolizumab as neoadjuvant therapy in progressive glioblastoma[81] and investigations of targeted therapies will undoubtedly continue to be pursued.

While many of the clinical trials for targeted therapy have been disappointing the recommendations in this updated guideline highlight the progress that has been made in identifying and interrogating key alterations in glioblastoma and the significant advancements that have been made in further understanding the heterogeneity within glioblastomas.

\section{Conclusions And Key Issues For Future Investigations}

Continuing work using whole genome, large-scale molecular studies, and methylation profiling continues to further elucidate the alterations that occur in glioblastomas over time and following therapy. Further understanding of the genetic landscape of infiltrating gliomas has already allowed us a greater understanding of the heterogeneity within these tumors and allowed us to begin integrating these molecular alterations into a classification system[30; 98-103] and targeted therapy. However, even within the current classification system there remains extensive molecular and behavioral diversity. An update of the WHO classification of Tumors the Central Nervous System is expected to be released later this year which is expected to further integrate molecular features into the classification system as has been evident in the c-IMPACT now publications.[18; 19; 98; 104; 105] This observation has been reinforced in 
recent clinical trials where molecular characteristics were not part of the eligibility criteria and yet the overall results have been disappointing with only a minority of patients demonstrating a meaningful response. Identification of useful biomarkers that predict response or resistance for these targeted therapeutic agents will be critical for further progress.

One of the major hindrances within studies related to progressive glioblastoma is that patients who are candidates for re-resection are often younger and healthier patients with a definable mass. Tissue from these cases may not reflect alterations present in more aggressive diffuse glioblastomas and further work to identify what alterations are present in these tumors will need to be undertaken.

\section{Abbreviations}

BEV

Bevacizumab

CNS

central nervous system

EGFR

epidermal growth factor receptor

GBM

glioblastoma

IHC

immunohistochemistry

MGMT

O6-methylguanine-methyltransferase

MMR

mismatch repair

NGS

next-generation sequencing

OS

overall survival

PD-1

programmed cell death protein

PD-L1

programmed death-ligand 1

PFS

progression free survival

Pts

patients

RT

radiotherapy

TMZ

temozolomide 
WHO

World Health Organization

\section{Declarations}

\section{Conflict of Interest (COI)}

All Guideline Task Force members were required to disclose all potential COls prior to beginning work on the guideline, using the COI disclosure form of the AANS/CNS Joint Guidelines Review Committee. The CNS Guidelines Committee and Guideline Task Force Chair reviewed the disclosures and either approved or disapproved the nomination and participation on the task force. The CNS Guidelines Committee and Guideline Task Force Chair may approve nominations of task force members with possible conflicts and restrict the writing, reviewing, and/or voting privileges of that person to topics that are unrelated to the possible COls. The authors have no personal, financial, or institutional interest in any of the drugs, materials, or devices described in this series of articles.

\section{Data transparency}

The author has ensured all data and materials as well as software applications or custom code supports their published claims and comply with field standards.

\section{Author Contributions}

The author listed on this publication agrees with the content included and gives explicit consent to the submission of this publication. The author obtained consent from the responsible authorities at the institute/organization where the work has been carried out, before the work was submitted.

The author whose name appear on this submission:

1) made substantial contributions to the conception or design of the work; or the acquisition, analysis, or interpretation of data; or the creation of new software used in the work;

2) drafted the work or revised it critically for important intellectual content;

3) approved the version to be published; and

4) agreed to be accountable for all aspects of the work in ensuring that questions related to the accuracy or integrity of any part of the work are appropriately investigated and resolved.

\section{Compliance with Ethical Standards}

Funding: These guidelines were funded exclusively by the Congress of Neurological Surgery and the Joint Section on Tumors of the Congress of Neurological Surgeons and the American Association of 
Neurological Surgeons, which received no funding from any outside commercial sources to support the development of this document.

\section{Ethical Approval}

This article does not contain any studies with human participants performed by any of the authors.

\section{Data Availability}

The data generated during and/or analyzed during the current study are available via www.cns.org/guidelines.

\section{Disclosures}

These guidelines were funded exclusively by the Congress of Neurological Surgery and the Joint Section on Tumors of the Congress of Neurological Surgeons and the American Association of Neurological Surgeons, which received no funding from any outside commercial sources to support the development of this document.

\section{Disclaimer of Liability}

This clinical systematic review and evidence-based guideline was developed by a multidisciplinary physician volunteer task force and serves as an educational tool designed to provide an accurate review of the subject matter covered. These guidelines are disseminated with the understanding that the recommendations by the authors and consultants who have collaborated in their development are not meant to replace the individualized care and treatment advice from a patient's physician(s). If medical advice or assistance is required, the services of a competent physician should be sought. The proposals contained in these guidelines may not be suitable for use in all circumstances. The choice to implement any particular recommendation contained in these guidelines must be made by a managing physician in light of the situation in each particular patient and on the basis of existing resources.

\section{Acknowledgements}

The guidelines task force would like to acknowledge the Congress of Neurological Surgeons Guidelines Committee for their contributions throughout the development of the guideline, and the American Association of Neurological Surgeons/Congress of Neurological Surgeons Joint Guidelines Review Committee for their review, comments, and suggestions throughout peer review, as well as the contributions of Trish Rehring, MPH, CHES, Senior Manager of Clinical Practice Guidelines for the CNS, and Mary Bodach, MLIS, from the Congress of Neurological Surgeons Guidelines Office for organizational assistance and reference librarian services, respectively as well as Jeremy Kupsco, PhD, Informationist, Emory University, for their valuable input as Medical Research Librarians. Throughout the review process, the reviewers and authors were blinded from one another. At this time the guidelines task force would like to acknowledge the following individual peer reviewers for their contributions: John O'Toole, MD, Brian 
Howard, MD, Jamie Van Gompel, MD, Howard Silberstein, MD, Navid Redjal, MD and Shawn HerveyJumper, MD.

\section{References}

1. Wen PY, Kesari S (2008) Malignant Gliomas in Adults. N Engl J Med 359:492-507

2. Thakkar JP, Dolecek TA, Horbinski C et al (2014) Epidemiologic and molecular prognostic review of glioblastoma. Cancer Epidemiol Biomarkers Prev 23:1985-1996

3. Stupp R, Mason WP, van den Bent MJ et al (2005) Radiotherapy plus Concomitant and Adjuvant Temozolomide for Glioblastoma. N Engl J Med 352:987-996

4. Choi SW, Shin H, Sa JK et al (2018) Identification of transcriptome signature for predicting clinical response to bevacizumab in recurrent glioblastoma. Cancer Med 7:1774-1783

5. Bagley SJ, Schwab RD, Nelson E et al (2019) Histopathologic quantification of viable tumor versus treatment effect in surgically resected recurrent glioblastoma. J Neurooncol 141:421-429

6. Weller M, Cloughesy T, Perry JR, Wick W (2012) Standards of care for treatment of recurrent glioblastoma-are we there yet? Neurooncology 15:4-27

7. Montemurro N, Perrini P, Blanco MO, Vannozzi R (2016) Second surgery for recurrent glioblastoma: A concise overview of the current literature. Clin Neurol Neurosurg 142:60-64

8. Brat DJ, Ryken TC, Kalkanis SN, Olson JJ (2014) The role of neuropathology in the management of progressive glioblastoma: a systematic review and evidence-based clinical practice guideline. $\mathrm{J}$ Neurooncol 118:461-478

9. Dalle Ore CL, Chandra A, Rick J et al (2018) Presence of Histopathological Treatment Effects at Resection of Recurrent Glioblastoma: Incidence and Effect on Outcome. Neurosurgery 85:793-800

10. Hu LS, Eschbacher JM, Heiserman JE et al (2012) Reevaluating the imaging definition of tumor progression: perfusion MRI quantifies recurrent glioblastoma tumor fraction, pseudoprogression, and radiation necrosis to predict survival. Neurooncology 14:919-930

11. Burger PC, Nelson JS, Boyko OB (1998) Diagnostic synergy in radiology and surgical neuropathology: radiographic findings of specific pathologic entities. Arch Pathol Lab Med 122:620632

12. Louis D, Ohgaki H, Wiestler O, WK C (2016) World Health Organization Histological Classification of Tumours of the Central Nervous System, Revised 4th edn. International Agency for Research on Cancer, France

13. Olson JJ, Fadul CE, Brat DJ, Mukundan S, Ryken TC (2009) Management of newly diagnosed glioblastoma: guidelines development, value and application. J Neurooncol 93:1

14. Holdhoff M, Ye X, Piotrowski AF et al (2019) The consistency of neuropathological diagnoses in patients undergoing surgery for suspected recurrence of glioblastoma. J Neurooncol 141:347-354

15. Azoulay M, Santos F, Shenouda G et al (2017) Benefit of re-operation and salvage therapies for recurrent glioblastoma multiforme: results from a single institution. J Neurooncol 132:419-426 
16. Woodworth GF, Garzon-Muvdi T, Ye X, Blakeley JO, Weingart JD, Burger PC (2013) Histopathological correlates with survival in reoperated glioblastomas. J Neurooncol 113:485-493

17. Okita Y, Narita Y, Miyakita $Y$ et al (2012) Pathological findings and prognostic factors in recurrent glioblastomas. Brain Tumor Pathol 29:192-200

18. Brat DJ, Aldape K, Colman H et al (2018) cIMPACT-NOW update 3: recommended diagnostic criteria for "Diffuse astrocytic glioma, IDH-wildtype, with molecular features of glioblastoma, WHO grade IV". Acta Neuropathol 136:805-810

19. Brat DJ, Aldape K, Colman H et al (2020) CIMPACT-NOW update 5: recommended grading criteria and terminologies for IDH-mutant astrocytomas. Acta Neuropathol 139:603-608

20. Pisapia DJ (2017) The Updated World Health Organization Glioma Classification: Cellular and Molecular Origins of Adult Infiltrating Gliomas. Arch Pathol Lab Med 141:1633-1645

21. Cohen MH, Shen YL, Keegan P, Pazdur R (2009) FDA Drug Approval Summary: Bevacizumab (Avastin ${ }^{\circledR}$ ) as Treatment of Recurrent Glioblastoma Multiforme. Oncologist 14:1131-1138

22. Kanamori M, Kikuchi A, Watanabe M et al (2014) Rapid and sensitive intraoperative detection of mutations in the isocitrate dehydrogenase 1 and 2 genes during surgery for glioma. 120:1288

23. Millward CP, Brodbelt AR, Haylock B et al (2016) The impact of MGMT methylation and IDH-1 mutation on long-term outcome for glioblastoma treated with chemoradiotherapy. Acta Neurochir 158:1943-1953

24. Johnson BE, Mazor T, Hong C et al (2014) Mutational Analysis Reveals the Origin and TherapyDriven Evolution of Recurrent Glioma. Science 343:189-193

25. Bai H, Harmancı AS, Erson-Omay EZ et al (2016) Integrated genomic characterization of IDH1-mutant glioma malignant progression. Nat Genet 48:59-66

26. Huang LE (2019) Friend or foe-IDH1 mutations in glioma 10 years on. Carcinogenesis 40:12991307

27. Liu B, Pang B, Liu H et al (2015) High mobility group A1 expression shows negative correlation with recurrence time in patients with glioblastoma multiforme. Pathology - Research and Practice 211:596-600

28. Buckner J, Giannini C, Eckel-Passow J et al (2017) Management of diffuse low-grade gliomas in adults - use of molecular diagnostics. Nature Reviews Neurology 13:340-351

29. Watanabe T, Nobusawa S, Kleihues P, Ohgaki H (2009) IDH1 Mutations Are Early Events in the Development of Astrocytomas and Oligodendrogliomas. Am J Pathol 174:1149-1153

30. Yan H, Parsons DW, Jin G et al (2009) IDH1 and IDH2 Mutations in Gliomas. N Engl J Med 360:765773

31. Mazor T, Chesnelong C, Pankov A et al (2017) Clonal expansion and epigenetic reprogramming following deletion or amplification of mutant IDH1. Proceedings of the National Academy of Sciences 114:10743-10748 
32. Pusch S, Sahm F, Meyer J, Mittelbronn M, Hartmann C, von Deimling A (2011) Scientific correspondence. Neuropathol Appl Neurobiol 37:428-430

33. Favero F, McGranahan N, Salm M et al (2015) Glioblastoma adaptation traced through decline of an IDH1 clonal driver and macro-evolution of a double-minute chromosome. Ann Oncol 26:880-887

34. JOHANNESSEN LE, BRANDAL P, MYKLEBUST T, MICCI HEIMS, PANAGOPOULOS F I (2018) MGMT Gene Promoter Methylation Status - Assessment of Two Pyrosequencing Kits and Three Methylation-specific PCR Methods for their Predictive Capacity in Glioblastomas. Cancer Genomics Proteomics 15:437-446

35. Hegi ME, Rajakannu P, Weller M (2012) Epidermal growth factor receptor: a re-emerging target in glioblastoma. Curr Opin Neurol 25:774-779

36. Brandes AA, Franceschi E, Paccapelo A et al (2017) Role of MGMT Methylation Status at Time of Diagnosis and Recurrence for Patients with Glioblastoma: Clinical Implications. Oncologist 22:432437

37. Agarwal S, Sharma MC, Jha P et al (2013) Comparative study of IDH1 mutations in gliomas by immunohistochemistry and DNA sequencing. Neurooncology 15:718-726

38. Hsu C-Y, Ho H-L, Lin S-C et al (2017) Comparative Assessment of 4 Methods to Analyze MGMT Status in a Series of 121 Glioblastoma Patients. Applied Immunohistochemistry \& Molecular Morphology 25:497-504

39. Kristensen LS, Michaelsen SR, Dyrbye H et al (2016) Assessment of Quantitative and Allelic MGMT Methylation Patterns as a Prognostic Marker in Glioblastoma. Journal of Neuropathology \& Experimental Neurology 75:246-255

40. Quillien V, Lavenu A, Ducray F et al (2016) Validation of the high-performance of pyrosequencing for clinical MGMT testing on a cohort of glioblastoma patients from a prospective dedicated multicentric trial. Oncotarget 7:61916-61929

41. Radke J, Koch A, Pritsch F et al (2019) Predictive MGMT status in a homogeneous cohort of IDH wildtype glioblastoma patients. Acta Neuropathologica Communications 7:89

42. Tanboon J, Williams EA, Louis DN (2015) The Diagnostic Use of Immunohistochemical Surrogates for Signature Molecular Genetic Alterations in Gliomas. Journal of Neuropathology \& Experimental Neurology 75:4-18

43. Mansouri A, Hachem LD, Mansouri S et al (2018) MGMT promoter methylation status testing to guide therapy for glioblastoma: refining the approach based on emerging evidence and current challenges. Neurooncology 21:167-178

44. Napoleoni L, Cortellini A, Cannita K et al (2019) Looking for A Place for Dose-Dense TMZ Regimens in GBM Patients: An Experience with MGMT Exploratory Evaluation. Bioengineering (Basel, Switzerland) 6:11

45. Ohgaki H, Kleihues P (2007) Genetic Pathways to Primary and Secondary Glioblastoma. Am J Pathol 170:1445-1453 
46. Hovinga KE, McCrea HJ, Brennan C et al (2019) EGFR amplification and classical subtype are associated with a poor response to bevacizumab in recurrent glioblastoma. J Neurooncol 142:337345

47. Lv S, Teugels E, Sadones J et al (2012) Correlation of EGFR, IDH1 and PTEN status with the outcome of patients with recurrent glioblastoma treated in a phase II clinical trial with the EGFR-blocking monoclonal antibody cetuximab. Int J Oncol 41:1029-1035

48. Olar A, Aldape KD (2014) Using the molecular classification of glioblastoma to inform personalized treatment. J Pathol 232:165-177

49. Zhang H, Berezov A, Wang Q et al (2007) ErbB receptors: from oncogenes to targeted cancer therapies. J Clin Investig 117:2051-2058

50. Brennan C, Momota H, Hambardzumyan D et al (2009) Glioblastoma subclasses can be defined by activity among signal transduction pathways and associated genomic alterations. PLoS ONE 4:e7752

51. Cioca A, Olteanu EG, Gisca MD, Morosanu CO, Marin I, Florian IS (2016) Expression of EGFR in Paired New and Recurrent Glioblastomas. Asian Pac J Cancer Prev 17:4205-4208

52. Binder DC, Ladomersky E, Lenzen A et al (2018) Lessons learned from rindopepimut treatment in patients with EGFRvIII-expressing glioblastoma. Translational Cancer Research:S510-S513

53. Pelloski CE, Ballman KV, Furth AF et al (2007) Epidermal Growth Factor Receptor Variant III Status Defines Clinically Distinct Subtypes of Glioblastoma. J Clin Oncol 25:2288-2294

54. Sugawa N, Ekstrand AJ, James CD, Collins VP (1990) Identical splicing of aberrant epidermal growth factor receptor transcripts from amplified rearranged genes in human glioblastomas. Proc Natl Acad Sci USA 87:8602-8606

55. Ekstrand AJ, Sugawa N, James CD, Collins VP (1992) Amplified and rearranged epidermal growth factor receptor genes in human glioblastomas reveal deletions of sequences encoding portions of the N- and/or C-terminal tails. Proc Natl Acad Sci USA 89:4309-4313

56. Lassman AB, van den Bent MJ, Gan HK et al (2018) Safety and efficacy of depatuxizumab mafodotin + temozolomide in patients with EGFR-amplified, recurrent glioblastoma: results from an international phase I multicenter trial. Neurooncology 21:106-114

57. Sepúlveda-Sánchez JM, Vaz M, Balañá C et al (2017) Phase II trial of dacomitinib, a pan-human EGFR tyrosine kinase inhibitor, in recurrent glioblastoma patients with EGFR amplification. Neurooncology 19:1522-1531

58. Lassman AB, Pugh SL, Gilbert MR et al (2015) Phase 2 trial of dasatinib in target-selected patients with recurrent glioblastoma (RTOG 0627). Neurooncology 17:992-998

59. Westphal M, Maire CL, Lamszus K (2017) EGFR as a Target for Glioblastoma Treatment: An Unfulfilled Promise. CNS Drugs 31:723-735

60. van den Bent MJ, Gao Y, Kerkhof M et al (2015) Changes in the EGFR amplification and EGFRvIII expression between paired primary and recurrent glioblastomas. Neurooncology 17:935-941 
61. D'Alessandris QG, Montano N, Cenci T et al (2013) Targeted therapy with bevacizumab and erlotinib tailored to the molecular profile of patients with recurrent glioblastoma. Preliminary experience. Acta Neurochir 155:33-40

62. Kamiya-Matsuoka C, Gilbert MR (2015) Treating recurrent glioblastoma: an update. CNS Oncology 4:91-104

63. Lau D, Magill ST, Aghi MK (2014) Molecularly targeted therapies for recurrent glioblastoma: current and future targets. 37:E15

64. Byron SA, Tran NL, Halperin RF et al (2018) Prospective Feasibility Trial for Genomics-Informed Treatment in Recurrent and Progressive Glioblastoma. Clin Cancer Res 24:295-305

65. Li R, Chen X, You Y et al (2015) Comprehensive portrait of recurrent glioblastoma multiforme in molecular and clinical characteristics. Oncotarget 6

66. Neilsen BK, Sleightholm R, McComb R et al (2019) Comprehensive genetic alteration profiling in primary and recurrent glioblastoma. J Neurooncol 142:111-118

67. Cimino PJ, McFerrin L, Wirsching H-G et al (2018) Copy number profiling across glioblastoma populations has implications for clinical trial design. Neurooncology 20:1368-1373

68. Wood MD, Reis GF, Reuss DE, Phillips JJ (2016) Protein Analysis of Glioblastoma Primary and Posttreatment Pairs Suggests a Mesenchymal Shift at Recurrence. Journal of Neuropathology \& Experimental Neurology 75:925-935

69. KOLODZIEJ MA, WEISCHER C, REINGES MHT et al (2016) NDRG2 and NDRG4 Expression Is Altered in Glioblastoma and Influences Survival in Patients with MGMT-methylated Tumors. Anticancer Res 36:887-897

70. Narsia N, Ramagiri P, Ehrmann J, Kolar Z (2017) Transcriptome analysis reveals distinct gene expression profiles in astrocytoma grades II-IV. Biomed Pap Med Fac Univ Palacky Olomouc Czech Repub 161:261-271

71. Heynckes S, Gaebelein A, Haaker G et al (2017) Expression differences of programmed death ligand 1 in de-novo and recurrent glioblastoma multiforme. Oncotarget 8:74170-74177

72. Berghoff AS, Kiesel B, Widhalm G et al (2014) Programmed death ligand 1 expression and tumorinfiltrating lymphocytes in glioblastoma. Neurooncology 17:1064-1075

73. Hodges TR, Ott M, Xiu J et al (2017) Mutational burden, immune checkpoint expression, and mismatch repair in glioma: implications for immune checkpoint immunotherapy. Neurooncology 19:1047-1057

74. Chen RQ, Liu F, Qiu XY, Chen XQ (2019) The Prognostic and Therapeutic Value of PD-L1 in Glioma. Front Pharmacol 9:1503-1503

75. Chen L, Han X, Anti -PD-1/PD-L1 therapy of human cancer: past, present, and future. The Journal of Clinical Investigation125:3384-3391

76. Rahman M, Kresak J, Yang C et al (2018) Analysis of immunobiologic markers in primary and recurrent glioblastoma. J Neurooncol 137:249-257 
77. Omuro A, Vlahovic G, Lim M et al (2017) Nivolumab with or without ipilimumab in patients with recurrent glioblastoma: results from exploratory phase I cohorts of CheckMate 143. Neurooncology 20:674-686

78. Reardon DA, Omuro A, Brandes AA et al (2017) OS10.3 Randomized Phase 3 Study Evaluating the Efficacy and Safety of Nivolumab vs Bevacizumab in Patients With Recurrent Glioblastoma: CheckMate 143. 19:iii21-iii21. Neuro-oncology

79. Filley AC, Henriquez M, Dey M (2017) Recurrent glioma clinical trial, CheckMate-143: the game is not over yet. Oncotarget 8:91779-91794

80. Cloughesy TF, Mochizuki AY, Orpilla JR et al (2019) Neoadjuvant anti-PD-1 immunotherapy promotes a survival benefit with intratumoral and systemic immune responses in recurrent glioblastoma. Nat Med 25:477-486

81. Daniel P, Sabri S, Chaddad A et al (2019) Temozolomide Induced Hypermutation in Glioma: Evolutionary Mechanisms and Therapeutic Opportunities. Frontiers in Oncology 9

82. McCord M, Steffens A, Javier R, Kam K-L, McCortney K, Horbinski C (2020) The efficacy of DNA mismatch repair enzyme immunohistochemistry as a screening test for hypermutated gliomas. Acta Neuropathologica Communications 8:15

83. Indraccolo S, Lombardi G, Fassan M et al (2019) Genetic, Epigenetic, and Immunologic Profiling of MMR-Deficient Relapsed Glioblastoma. Clin Cancer Res 25:1828-1837

84. Lamszus K, Ulbricht U, Matschke J, Brockmann MA, Fillbrandt R, Westphal M (2003) Levels of Soluble Vascular Endothelial Growth Factor (VEGF) Receptor 1 in Astrocytic Tumors and Its Relation to Malignancy, Vascularity, and VEGF-A. Clin Cancer Res 9:1399-1405

85. de Groot JF, Fuller G, Kumar AJ et al (2010) Tumor invasion after treatment of glioblastoma with bevacizumab: radiographic and pathologic correlation in humans and mice. Neurooncology 12:233242

86. Bloch O, Safaee M, Sun MZ et al (2013) Disseminated progression of glioblastoma after treatment with bevacizumab. Clin Neurol Neurosurg 115:1795-1801

87. Erdem-Eraslan L, van den Bent MJ, Hoogstrate $Y$ et al (2016) Identification of Patients with Recurrent Glioblastoma Who May Benefit from Combined Bevacizumab and CCNU Therapy: A Report from the BELOB Trial. Cancer Res 76:525-534

88. Cardona AF, Rojas L, Wills B et al (2019) A comprehensive analysis of factors related to carmustine/bevacizumab response in recurrent glioblastoma. Clin Transl Oncol 21:1364-1373

89. Boisen MK, Holst CB, Consalvo N, Chinot OL, Johansen JS (2017) Plasma YKL-40 as a biomarker for bevacizumab efficacy in patients with newly diagnosed glioblastoma in the phase 3 randomized AVAglio trial. Oncotarget 9:6752-6762

90. Kessler T (2016) Predicting glioblastoma response to bevacizumab through marker profiling? Neurooncology 18:149-150

91. Castro BA, Aghi MK (2014) Bevacizumab for glioblastoma: current indications, surgical implications, and future directions. NeuroSurg Focus 37:E9-E9 
92. Romani M, Pistillo MP, Carosio R, Morabito A, Banelli B (2018) Immune Checkpoints and Innovative Therapies in Glioblastoma. Front Oncol 8:464-464

93. Brahm CG, van Linde ME, Enting RH et al (2020) The Current Status of Immune Checkpoint Inhibitors in Neuro-Oncology: A Systematic Review. Cancers 12:586

94. Reardon DA, Brandes AA, Omuro A et al (2020) Effect of Nivolumab vs Bevacizumab in Patients With Recurrent Glioblastoma: The CheckMate 143 Phase 3 Randomized Clinical Trial. JAMA oncology $6: 1-8$

95. Louis DN, Giannini C, Capper D et al (2018) CIMPACT-NOW update 2: diagnostic clarifications for diffuse midline glioma, H3 K27M-mutant and diffuse astrocytoma/anaplastic astrocytoma, IDHmutant. Acta Neuropathol 135:639-642

96. Shirahata M, Iwao-Koizumi K, Saito S et al (2007) Gene Expression-Based Molecular Diagnostic System for Malignant Gliomas Is Superior to Histological Diagnosis. Clin Cancer Res 13:7341-7356

97. Eckel-Passow JE, Lachance DH, Molinaro AM et al (2015) Glioma Groups Based on 1p/19q, IDH, and TERT Promoter Mutations in Tumors. N Engl J Med 372:2499-2508

98. Hirose Y, Sasaki H, Abe M et al (2013) Subgrouping of gliomas on the basis of genetic profiles. Brain Tumor Pathol 30:203-208

99. Weller M, Weber RG, Willscher E et al (2015) Molecular classification of diffuse cerebral WHO grade II/III gliomas using genome- and transcriptome-wide profiling improves stratification of prognostically distinct patient groups. Acta Neuropathol 129:679-693

100. Stichel D, Ebrahimi A, Reuss D et al (2018) Distribution of EGFR amplification, combined chromosome 7 gain and chromosome 10 loss, and TERT promoter mutation in brain tumors and their potential for the reclassification of IDHwt astrocytoma to glioblastoma. Acta Neuropathol 136:793-803

101. Louis DN, Wesseling P, Aldape K et al (2020) cIMPACT-NOW update 6: new entity and diagnostic principle recommendations of the cIMPACT-Utrecht meeting on future CNS tumor classification and grading. Brain Pathol 30:844-856

102. Ellison DW, Aldape KD, Capper D et al cIMPACT-NOW update 7: advancing the molecular classification of ependymal tumors. Brain Pathology $\mathrm{n} / \mathrm{a}$

103. 103. Stichel D, Ebrahimi A, Reuss D et al (2018) Distribution of EGFR amplification, combined chromosome 7 gain and chromosome 10 loss, and TERT promoter mutation in brain tumors and their potential for the reclassification of IDHwt astrocytoma to glioblastoma. Acta Neuropathologica 136:793-803

104. 104. Louis DN, Wesseling P, Aldape $\mathrm{K}$ et al (2020) cIMPACT-NOW update 6: new entity and diagnostic principle recommendations of the cIMPACT-Utrecht meeting on future CNS tumor classification and grading. Brain Pathology 30:844-856

105. 105. Ellison DW, Aldape KD, Capper D et al cIMPACT-NOW update 7: advancing the molecular classification of ependymal tumors. Brain Pathology n/a 


\section{Tables}

Table I. Neuropathologic techniques/ Radionecrosis in progressive glioblastoma

Abbreviations: BEV, Bevacizumab; ECOG PS, Eastern Cooperative Oncology Group performance status; GBM, glioblastoma; HR, hazard ratios; MGMT, 06-methylguanine-

methyltransferase; MRI, Magnetic resonance imaging; MVD, mean vascular density; OS, overall survival; pMRI-FTB, perfusion MRI-fractional tumor burden; PTRE, post treatment radiation effect; pts, patients; RT, radiation therapy; TMZ, temozolomide

Table II. MIB-1 quantification in progressive glioblastoma

Abbreviations: GBM, glioblastoma; OS, overall survival; pts, patients

Table III. Repeat IDH testing in progressive glioblastoma

Abbreviations: CNA, copy number abnormalities; GBM, glioblastoma; HMGA1, high mobility group A1; IHC, immunohistochemistry; PI3K, phosphoinositide 3-kinase; pts, patients; RTK, receptor tyrosine kinase

Table IV. Repeat MGMT promoter methylation testing in progressive glioblastoma

Abbreviations: dBiSeq, direct bisulfite sequencing; dd-TMZ, dose-dense temozolomide; FFPE, formalin fixed paraffin embedded; GBM, glioblastoma; GKS, gamma knife surgery; IHC, immunohistochemistry; MGMT, 06-methylguanine-methyltransferase; mo., months; mOS, median overall survival; mPFS, median progression free survival; MSP, methylation-specific polymerase chain reaction; msPCR, methylationspecific PCR;MST, median survival time; OS, overall survival; PCR, polymerase chain reaction; PFS, progression free survival time; PSQ, pyrosequencing; pts, patients; qMSP, quantitative Methylation-Specific PCR;RT, radiation therapy; SqMSP, semi-quantitative methylation specific PCR; TMZ, temozolomide; unmethyl, unmethylated; $\mathbf{w t}$, wild type

\section{Table V. EGFR amplification and mutational testing in progressive glioblastoma}

Abbreviations: BEV, bevacizumab; CR, complete response; ECD, extracellular domain EGFR, epidermal growth factor; EGFRvIII, epidermal growth factor receptor variant III; EGFRvIV, epidermal growth factor receptor variant IV; FISH, fluorescence in situ hybridization; GBM, glioblastoma;

IHC, immunohistochemistry; msPCR, methylation specific PCR; OS, overall survival; PCR, polymerase chain reaction; PFS, progression free survival; PR, partial response; P-SRC, phosphorylated$S R C$, pts, patients; RR, radiologic response; RT, radiation therapy; SNV, single nucleotide variant; TMZ, temozolomide; wt, wild type

Table VI. Whole genome or large panel sequencing in progressive glioblastoma 


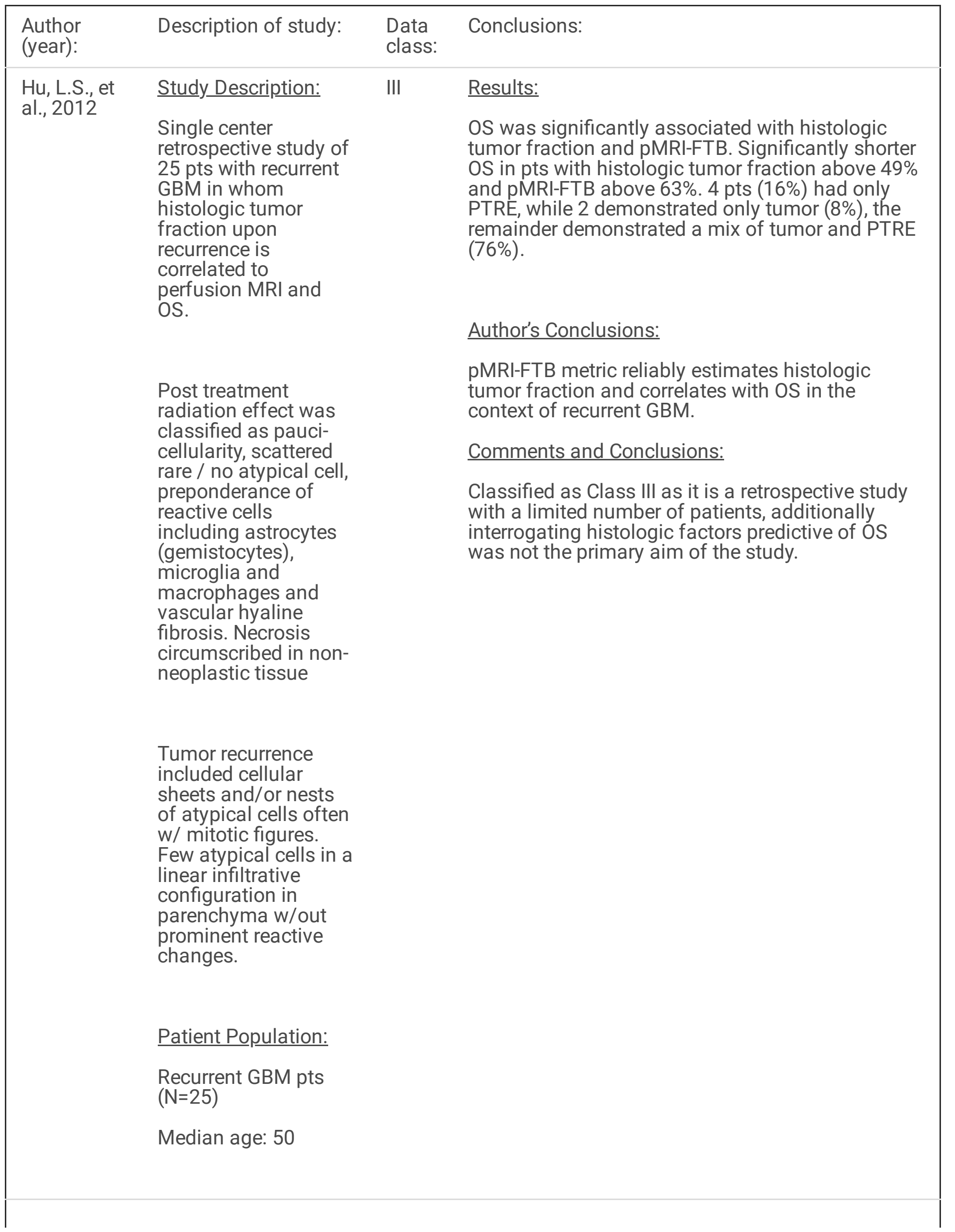


Woodworth, Study Description: G.F. et al., 2013

Single center retrospective study of 59 pts with recurrent GBM in whom pathology was reviewed for treatment related effects at the time of re-resection.

Treatment effect is defined as paucicellular coagulation necrosis, gliosis, pleomorphism in low density areas, and vascular changes including fibrinoid necrosis, telangiectasia, and hyalinization.

Active tumor was defined as highly cellular tumor similar to GBM prior to treatment, however, necrosis or MVP was required. Mitosis were usually present but not required in a small specimen.

Presence of only low grade tumor was not considered as "active tumor".
III

$\underline{\text { Results: }}$

$71 \%$ of pts had at least focal high grade glioma, with only $29 \%$ of pts with no evidence of active high grade tumor. Pathologic pseudoprogression at re-operation and gross total resection were associated with survival.

\section{Author's Conclusions:}

Histopathologic assessment of pseudoprogression can be used to prognostically categorize pts and histologic assessment is superior to standard radiologic assessment.

Comments and Conclusions:

Classified as Class III because study is a retrospective study that does not interrogate what features of "active tumor" are prognostic nor does it interrogate if their proposed definition is ideal.

Patient Population:

Recurrent GBM pts

$(\mathrm{N}=59)$

Median age at $d x: 53$

Azoulay, M et al., 2017
Study Description:

Single center retrospective study of 183 pts with recurrent GBM, 69 of whom underwent re-
III

Results:

Pathology at re-resection:

$85 \%$ of pts had residual GBM, $15 \%$ of pts had only RT induced necrosis, and $1 \mathrm{pt}$. had developed a sarcoma. Absence of residual GBM on repeat 
resection. Tumor

present on re-

resection histology

was correlated with

survival

Patient Population:

GBM patients $(\mathrm{n}=$

183)

Re-resection ( $\mathrm{n}=69)$

Median age: 58

pathology specimen (HR 0.23) were shown to affect survival.

\section{Author's Conclusions:}

Surgery allows for confirmation of histopathology when recurrence cannot be distinguished from radionecrosis based on imaging alone. Surgery at the time of progression leads to increased survival in this study and having more than one surgery following recurrence can significantly improve patient outcome.

\section{Comments and Conclusions:}

Classified as Class III as this is retrospective study in which histopathology was not the main focus, only $+/$ - for residual tumor was interrogated in this study.

Blumenthal, Study Description: III D.T., et al., 2018

Single center study of
15 pts treated $\mathrm{w} /$

TMZ + RT followed by

BEV. Re-resection after BEV samples were compared to the pts initial were evaluated for morphologic changes and mean vascular density (MVD).

Patient Population:

BEV treated patients $(n=15)$

Median age: 42 (2073)

BEV-naive $(\mathrm{n}=44)$

Median age: 68 (2477)

Holdhoff M., et al., 2018

\section{Results:}

No morphologic differences were apparent between pre- and post-BEV-treated tumor samples. There was a statistically significant decrease in mean vascular density overall between pre- and post $\mathrm{BEV}$ treated specimens (pooled, $\mathrm{p}<0.001$ ). No significant difference was identified when looking at the individual cases $(p=0.2)$, however, 7 patients did demonstrate a significant decrease in MVD.

\section{Author's Conclusions:}

No overt changes in histopathology were observed after antiangiogenic therapy, but mean vascular density was present decreased status post BEV treatment. No predictive or prognostic clinical or imaging value correlated with "response" to BEV as assessed by MVD.

Comments and Conclusions:

Classified as Class III because study is retrospective review with a limited number of specimens. No significant impact of BEV treatment on histology.

\section{Study Description:}

Study of variability between 48 pathologists (44 of which were neuropathologists) on
III

Results:

Agreement in overall assessment of disease activity within this survey, determined using Fleiss' kappa statistics was $0.228(95 \% \mathrm{Cl} 0.22-0.24)$. This is consistent only with "marginal agreement" between observers. 
differentiating active

tumor and inactive

tumor/treatment

effect in 13 cases of

recurrent

glioblastoma.

Author's Conclusions:

Formal criteria and terminology need to be developed to help improve consistency and reliability.

\section{Comments and Conclusions:}

Classified as Class III because study is a prospective study with determination of Kappa statistics, however, the cut-off points and delineations made by the authors limited the utility of the study and they either did not report or did not analyze all the data collected.

Bagley S.J. et al., 2018
Study Description:

Single institution retrospective study of 37 pts with recurrent GBM who underwent re-resection.

Tumor burden was assessed by extent of viable tumor, mitoses per 10 high-power fields, Ki-67 proliferative index.

Treatment related effects were categorized as vascular hyalinization, rarefaction, hemosiderin, and geographic necrosis.
III

Results:

Variables associated with OS in multivariate analysis included the Ki-67 proliferation index $(p=0.003)$, time from initial diagnosis to repeat surgery $(p=0.017)$, and ECOG PS (0.002). The extent of viable tumor showed a trend $(p=0.06)$ toward decreased OS on univariate analysis but not multivariate.

\section{Author's Conclusions:}

In patients with glioblastoma undergoing repeat resection following $T M Z+R T$, high $\mathrm{Ki}-67$ index in the recurrent specimen, short time to recurrence, and poor ECOG PS are independently associated with worse OS. Histopathologic quantification of viable tumor versus therapy-related changes were not associated with OS.

\section{Comments and Conclusions:}

Classified as Class III because study is retrospective review with a limited sample size, but did assess histologic findings on a scale (not $+/-$ ).

Patient Population:

Recurrent GBM pts ( $\mathrm{n}$ $=37$ )

Median age: 61

Dalle Ore, C.L., et al., 2019
Study Description:

Single center retrospective study of 110 pts with recurrent GBM status post TMZ
III

Results:

Treatment effects were noted in $54 \%$ of cases, with $49 \%$ of cases having tumor and treatment effect, and $5.4 \%$ of cases with only treatment effect. Sarcoma elements were present in $7 \%$ of pts. The 
+ RT, 60 pts also were treated with BEV in whom pathology was reviewed for treatment related effects at the time of re-resection.

Treatment related changes are characterized by coagulative necrosis, fibrinous vascular necrosis, and hyalinized vessels.

Patient Population:

Recurrent GBM pts $(\mathrm{N}=110)$

Median age at $1^{\text {st }}$ surgery: 54.9 presence of treatment effect was significantly associated with more recent RT therapy (8.9 mo. vs 13.8 mo., $p=0.003)$. Patients treated with BEV prior to reoperation were less likely to have treatment effect $(20 \%$ vs $65 \%$, P < .001). The presence of treatment effect was not correlated with MGMT promoter methylation status or IDH-1 mutation. Treatment effect was associated with increased survival after re-operation $(p=0.014)$ but not OS from primary resection $(p=0.172)$.

\section{Author's Conclusions:}

Treatment related changes are present in the majority of patients and is associated with earlier re-resection, improved survival from re-resection and unchanged OS from primary resection.

\section{Comments and Conclusions:}

Classified as Class III because study is a retrospective study and \% tumor/treatment effect was not reported for the majority of pt's nor considered in the statistics, however there is a large number of pts.

\begin{tabular}{|c|c|c|c|}
\hline $\begin{array}{l}\text { Author } \\
\text { (year): }\end{array}$ & Description of study: & $\begin{array}{l}\text { Data } \\
\text { class: }\end{array}$ & Conclusions: \\
\hline \multirow{5}{*}{$\begin{array}{l}\text { Okita, } \\
\text { Y., et } \\
\text { al., } \\
2012\end{array}$} & Study Description: & \multirow[t]{5}{*}{ III } & Results: \\
\hline & $\begin{array}{l}\text { Retrospective study of } 32 \\
\text { pts with recurrent GBM in } \\
\text { which prognostic factors } \\
\text { were assessed. }\end{array}$ & & $\begin{array}{l}\text { MIB-1 indices in recurrent tumors significantly } \\
\text { correlated with OS in multivariate analysis }(p=0.004) \text {. } \\
\text { Median survival time was not significantly } \\
\text { associated with MGMT promoter methylation } \\
(p=0.8) .6 / 18(33 \%) \text { pts showed a change in } \\
\text { methylation status upon recurrence. }\end{array}$ \\
\hline & $\begin{array}{l}\text { Therapy related changes } \\
\text { were defined as } \\
\text { pseudopalisading } \\
\text { necrosis, coagulation } \\
\text { necrosis, gemistocytic } \\
\text { cells, and giant cells. }\end{array}$ & & $\begin{array}{l}\text { Author's Conclusions: } \\
\text { Only MIB-1 index in recurrent GBM is a significant } \\
\text { prognostic factor. MGMT promoter methylation } \\
\text { status and degenerative changes in tumor cells in } \\
\text { recurrent tumors had no correlation with survival } \\
\text { time or PFS. }\end{array}$ \\
\hline & Patient Population: & & Comments and Conclusions: \\
\hline & $\begin{array}{l}\text { Recurrent GBM pts } \\
(\mathrm{N}=32) \\
\text { Median age: } 57(19-71)\end{array}$ & & $\begin{array}{l}\text { Classified as Class III as it is a retrospective study } \\
\text { with a limited sample size, histologic features were } \\
\text { examined only by }+/- \text { of particular findings of } \\
\text { treatment effect. }\end{array}$ \\
\hline
\end{tabular}

Abbreviations: EMT, epithelial mesenchymal transition; GA, genomic 


\begin{tabular}{|c|c|c|c|}
\hline $\begin{array}{l}\text { Author } \\
\text { (year): }\end{array}$ & $\begin{array}{l}\text { Description of } \\
\text { study: }\end{array}$ & $\begin{array}{l}\text { Data } \\
\text { class: }\end{array}$ & Conclusions: \\
\hline $\begin{array}{l}\text { Kanamori, } \\
\text { M., et al., } \\
2014\end{array}$ & $\begin{array}{l}\text { Study } \\
\text { Description: } \\
\text { Single center } \\
\text { prospective } \\
\text { study of the } \\
\text { use of } \\
\text { intraoperative } \\
\text { IDH mutation } \\
\text { analysis in } \\
\text { cases of } \\
\text { suspected non- } \\
\text { neoplastic } \\
\text { lesion (18), low } \\
\text { grade gliomas } \\
\text { (5), low grade } \\
\text { infiltrating } \\
\text { gliomas (3), } \\
\text { high grade } \\
\text { gliomas (10), } \\
\text { and radiation } \\
\text { necrosis (3). } \\
\\
\text { Patient } \\
\text { Population: } \\
\text { Use for } \\
\text { determining } \\
\text { residual tumor } \\
\text { in treatment } \\
\text { effect ( } \mathrm{N}=3)\end{array}$ & III & $\begin{array}{l}\text { Results: } \\
\text { IDHPCR was performed at the time of frozen section, could } \\
\text { be performed and resulted in } 60-65 \text { min, and had excellent } \\
\text { correspondence with IHC. Successfully used to determine } \\
\text { tumor necrosis vs residual tumor in } 3 \text { cases, in which tumor } \\
\text { was identified in all } 3 \text { cases, Detects an IDH mutation even } \\
\text { in cases of low tumor burden } \\
\text { Author's Conclusions: } \\
\text { Per prior report (Capper et al., 2010) glioma cells are present } \\
\text { in most cases of radiation necrosis, even when definitive } \\
\text { tumor is not present on histology. Thus, while this technique } \\
\text { is not needed for intraoperative purposes it can help } \\
\text { estimate the proportion of tumor cells in these specimens. } \\
\text { Comments and Conclusions: } \\
\text { Classified as Class III, while prospective, there is a limited } \\
\text { number of pts, particularly in the arm of study of most } \\
\text { interest for our review. }\end{array}$ \\
\hline $\begin{array}{l}\text { Johnson, } \\
\text { B., et al., } \\
2014\end{array}$ & $\begin{array}{l}\text { Study } \\
\text { Description: } \\
\text { Retrospective } \\
\text { study of } 23 \\
\text { IDH mutant } \\
\text { grade Il glioma, } \\
\text { favoring } \\
\text { astrocytoma, } \\
\text { and pts with } \\
\text { progressive } \\
\text { disease in } \\
\text { whom the } \\
\text { primary and } \\
\text { recurrent } \\
\text { specimen were } \\
\text { sequenced to } \\
\text { assess } \\
\text { mutational } \\
\text { differences. }\end{array}$ & III & $\begin{array}{l}\text { Results: } \\
\text { IDH mutation was retained in all } 23 \text { cases. 54\% of } \\
\text { mutations detected in the primary tumor were present upon } \\
\text { recurrence. IDH1, TP53, and ATRX were the most commonly } \\
\text { retained mutations. Of the } 10 \text { pts treated with TMZ } 6(60 \%) \\
\text { demonstrated a hypermutated phenotype upon progression } \\
\text { and progressed to glioblastoma. } 97 \% \text { of TMZ associated } \\
\text { mutations were C>T/G>A. Mutations involving the RB and } \\
\text { AKT-mTOR pathways were identified only in pts who } \\
\text { progressed to glioblastoma and none of the grade II-III } \\
\text { recurrences. In a subset of patients these mutations seem } \\
\text { to be linked to TMZ induced mutations. } \\
\text { Authors' Conclusions: } \\
\text { A significant proportion of mutations in primary gliomas are } \\
\text { lost at recurrence. TMZ-treated pts showed TMZ-induced }\end{array}$ \\
\hline
\end{tabular}

Page $33 / 76$ 
Patient

Population:

Paired

progressive

IDHmutant

glioma pts

$(\mathrm{N}=23)$

TMZ treated pts $(\mathrm{N}=10)$ mutagenesis and a proportion hypermutagenasis and appeared to follow an evolutionary path to high-grade glioma distinct from that in untreated patients.

\section{Comments and Conclusions:}

Classified as Class III as it is a retrospective study with a limited sample size and little reported demographic information. This study attempted to get predominantly astrocytomas but likely included some oligodendrogliomas.

Liu, B., et al., 2015

Study. Description:

Retrospective study of 25 recurrent high grade gliomas assessing expression of HMGA1 and

IDH in matched primary resection and recurrent GBM specimens.

HMGA1 and IDH-1 R132H expression were assessed by IHC.

Patient Population:

Recurrent GBM pts $(\mathrm{N}=25)$

Median age: 44.5 (30-62)

Bai, H., et al., 2016
Study.

Description:

Retrospective study of 41 progressive IDH1 mutant gliomas by
III

$\underline{\text { Results: }}$

HMGA1 overexpression was found significantly more in recurrent GBM $(15 / 25)$ than in initial GBM $(9 / 25 ; P=0.002)$, no correlation was identified between HMGA1 expression and adjuvant therapy $(P=0.516)$. For IDH-1 R132 mutation, 3 cases $(3 / 25,12 \%)$ were found in both initial and recurrent GBM groups. No correlation was identified between HMGA1 expression and IDH-1 R132H mutation in initial GBM group $(P=0.922)$. Nine pts who had initial GBM overexpressing HMGA1 had a median PFST of 7.3 months (95\% Cl: 2.1-13.2 months), while pts with initial GBM with little or no HMGA1 expression had a median survival time of 11.1 months (95\% Cl: 4.6-26.8 months; log-rank test: $P=$ 0.044

Author's conclusions:

No difference of IDH-1R132H mutations was found between initial and recurrent GBM patients. HMGA1 may be a promising target in the prognosis and treatment of GBMs, especially recurrent GBMs.

\section{Comments and conclusions:}

Classified as class III as it is a retrospective study with a limited number of pts. Conclusions about the role and expression of HMGA1 need to be further investigated.
Results:

Progressed tumors acquired an average of 21 mutations compared to the initial tumor and $27 \%$ of mutations and $30 \%$ of CNAs present in the initial tumor were no longer present in the progressed sample. IDH1 mutation was not lost in any of the paired tumors. $27 \%$ of progressive gliomas acquired different coding mutations in the same glioma 
exome sequencing of paired specimens from initial diagnosis and their progressed counterpart.

Patient

Population:

Progressive glioma pts

$(\mathrm{N}=41)$

Average age at initial resection: 38.9 (21-58) driver genes, such as TP53, ATRX, CIC and FUBP1, in which mutations were observed in their lower-grade counterparts.

During glioma progression, the receptor tyrosine kinase (RTK)-RAS-phosphoinositide 3-kinase (PI3K) pathway, the NOTCH pathway and FAT receptors were most frequently altered (in $22 \%, 12 \%$ and $10 \%$ of the patients, respectively). The most frequently amplified genomic region during progression was the MYC locus on chromosome $8 q$ (22\% of pts), and amplification was significantly associated with progression $(q=1.7 \times 10-3$, likelihood-ratio test)

\section{Author's Conclusions:}

During progression numerous convergent alterations occur, including activation of the MYC and RTK-RAS-PI3K signaling pathways, alterations in cell cycle regulators such as CDKN2A-CDKN2B, upregulation of FOXM1-and E2F2mediated cell cycle transitions, and epigenetic silencing of key developmental transcription factors.

\section{Comments and Conclusions:}

Classified as Class III as it is a retrospective study with a limited samples size. The cohort included oligodendrogliomas and not all tumors progressed to glioblastoma.

alterations; GBM, glioblastoma; GEO, Gene Expression Omnibus; GGN, German glioma network; KPS, Karnofsky Performance Status Scale; mRNA, messenger RNA; OS, overall survival; PI3K, Phosphoinositide 3-kinase; pts, patients; RT, radiation therapy; TCGA, The Cancer Genome Atlas; TMZ, temozolomide; TTP, time to progression

\section{Table VII. Immune checkpoint biomarker testing in progressive glioblastoma}

Abbreviations: BEV, bevacizumab; GBM, glioblastoma; G-CIMP, cytosine-phosphate-guanine (CpG) island methylator phenotype; IHC, immunohistochemistry; MGMT, 06-methylguaninemethyltransferase; MMR, mismatch repair; mo., months; mOS, median overall survival; mPFS, median progression free survival; mRNA, messenger RNA; MSI, microsatellite instability; NIV01+IPI3, nivolumab 1 $\mathrm{mg} / \mathrm{kg}$ + ipilimumab $3 \mathrm{mg} / \mathrm{kg}$ every 3 weeks (Q3W) for 4 doses, then nivolumab $3 \mathrm{mg} / \mathrm{kg}$ Q2W; NIV03, nivolumab 3 mg/kg every 2 weeks; NIVO3+IPI1, nivolumab $3 \mathrm{mg} / \mathrm{kg}+$ ipilimumab $1 \mathrm{mg} / \mathrm{kg}$ Q3W for 4 doses, then nivolumab $3 \mathrm{mg} / \mathrm{kg}$ Q2W; OS, overall survival; PD-L1, programmed death-ligand 1; PFS, progression free survival; pts, patients; qRT-PCR, Real-time quantitative PCR ; RT, radiation therapy; TIL, tumor infiltrating lymphocytes; TMB, tumor mutational burden; TMZ, temozolomide

Table VIII. Bevacizumab biomarker testing in progressive glioblastoma

Abbreviations: GBM, glioblastoma; pts, patients; BEV, bevacizumab; FTM, fotemustine; RR, radiologic 


$\begin{array}{llll}\text { Author (year): } & \text { Description of study: } & \begin{array}{l}\text { Data } \\ \text { class: }\end{array}\end{array}$

Okita, Y., et al., 2012
Study Description:

Retrospective study of 32 pts with recurrent GBM in which prognostic factors were assessed.

Therapy related changes were defined as pseudopalisading necrosis, coagulation necrosis, gemistocytic cells, and giant cells.

Patient Population:

Recurrent GBM pts $(\mathrm{N}=32)$

Median age: 57 (19-71)

III

\section{Results:}

MIB-1 indices in recurrent tumors significantly correlated with OS in multivariate analysis $(p=0.004)$. MST was not significantly associated with MGMT promoter methylation $(p=0.8) .6 / 18(33 \%)$ pts showed a change in methylation status upon recurrence.

\section{Author's Conclusions:}

Only MIB-1 index in recurrent GBM is a significant prognostic factor. MGMT promoter methylation status and degenerative changes in tumor cells in recurrent tumors had no correlation with survival time or PFS.

\section{Comments and Conclusions:}

Classified as Class III as it is a retrospective study with a limited sample size, histologic features were examined only by $+/$ - of particular findings of treatment effect.

Yang, P., et al., 2015

\section{Study Description:}

Prospective study of 274 pts with GBM looking at the effect IDH mutation and MGMT promoter methylation on treatment.

\section{Patient Population:}

GBM pts $(\mathrm{N}=274)$

Median age: 48 (18-81)

\section{II}

\section{Results:}

IDH mutations was observed in 56 cases $(21 \%)$ of cases and MGMT promoter methylation was observed in 95 cases (40\%). The IDH1 and MGMT promoter methylation status of the study populations were: 32 (14\%) IDH mutant and methylated MGMT promoter, 54 (25\%) wild type IDH and methylated MGMT promoter, 15 (7\%) IDH mutant and unmethylated MGMT promoter pts, and $128(56 \%)$ wild type IDH and unmethylated MGMT promoter pts.

TMZ and Radiation Therapy, OS and PFS was most favorable for those with tumors harboring both $I D H$ mutant and methylated

MGMT promoter (median OS: 35.8 mo., median PFS: 27.5 mo.); either IDH mutant or methylated MGMT promoter exhibited intermediate OS and PFS (mOS: 36 and 17.1 mo.; mPFS: 12.2 mo. and 9.9 mo., respectively); poorest OS and PFS was observed in wild type IDH, MGMT promoter unmethylated (mOS: 15 mo., mPFS: 9.7 mo.).

\section{Author's Conclusions:}


IDH mutation and MGMT promoter methylation status are independently associated with

favorable outcome in TMZ+RT treated GBM and have distinct biologic functions. TMZ shows the greatest efficacy in wild type $I D H$ tumors.

\section{Comments and Conclusions:}

Classified as Class II as it is a prospective study with a relatively large sample size in which multivariate analysis was performed.

Kristensen, L.S., et al., 2016
Study Description:

Retrospective study of 151 primary GBM pts treated by the Stupp protocol in which different methods of MGMT methylation status was assessed. Survival analysis was also performed.

\section{Patient Population:}

Primary GBM pts $(n=151)$

\section{Results:}

$34 \%$ (51) of pts were methylation positive by qMSP, of which the mean methylation level was $15.2 \%$ and median was $2.4 \%$.

$36 \%$ (47) of pts were methylated (>9\%) by standard pyrosequencing, with mean and median methylation levels of $37.3 \%$ and $30 \%$ respectively.

There is a strong association between the methods and methylation levels $(p<0.001$, $\mathrm{p}=0.002$ ).

Methylation by qMSP and pyrosequencing and IHC was strongly associated $(p=0.009$ and $\mathrm{P}<0.001$ respectively), IHC was positive in $43.9 \%$ and $48.8 \%$ of qMSP and pyrosequencing unmethylated samples, and negative in the majority of methylated $78 \%$ and $84.4 \%$ respectively.

All 3 methods correlate well with OS qMSP $(p=0.006)$, pyrosequencing $(p=0.002)$, and $\mathrm{IHC}$ $(p=0.009)$.

MGMT promoter methylation was associated with better OS ( $p=0.006$; qMSP and $p=0.002$; standard pyrosequencing), and the presence of the protein was associated with worse OS ( $p=$ 0.009 ).

\section{Author's Conclusions:}

Use of both MGMT promoter methylation and MGMT IHC but not allelic methylation data as prognostic markers in patients with TMZ-treated glioblastoma.

Comments and Conclusions:

Classified as Class III because it is a retrospective study with a relatively large 
number of specimens, however, factors other than methylation status were not evaluated.

Millward, C.P., Study Description: et al., 2016
III

Retrospective study of $100 \mathrm{GBM}$ pts to assess for a correlation with MGMT promoter methylation status and IDH mutation response to chemo radiotherapy.

\section{Patient Population:}

Primary GBM pts $(\mathrm{N}=100)$

\section{IDH mutant ( $\mathrm{N}=5)$}

Median age at dx: 54 (18-68)

$53 \%$ had MGMT promoter methylation $>9 \%$

Results:

Independent prognostic variables for OS and PFS were female sex (only for OS, $p=0.019$ ), MGMT promoter methylation ( $p<0.0001$, $\mathrm{p}=0.001$, respectively), and IDH mutation $(p=0.023, p=0.018$, respectively).

Kaplan-Meier survival analysis showed that MGMT methylated/IDH1 mutant gliomas were associated with a significantly longer OS 66.8 months and PFS 16.9 months when compared with MGMT methylated/IDH1 wild type gliomas (OS 15.5 months and PFS 9.4 months) and MGMT unmethylated/IDH1 wild type gliomas (OS 11.1 months and PFS 6.3 months) ( $p=$ 0.000).

\section{Author's Conclusions:}

Combination of MGMT promoter methylated/IDH1 mutant glioma is associated with considerably longer OS and PFS in this series of chemoradiotherapy-treated glioblastoma tumors.

\section{Comments and Conclusions:}

Classified as Class III as it is a retrospective study with a limited sample size.

Quillien, V., et al. 2016
Study Description:

Prospective multiinstitution trail in which analysis of the MGMT promoter methylation status by pyrosequencing and methylation-specific PCR on frozen section tissue and formalinfixed paraffinembedded tissue from 139 GBM patients and the correlation of PFS and OS with specific cut-off thresholds.

MGMT promoter methylation was assessed by pyrosequencing and $\underline{\text { Results: }}$

MGMT promoter methylation as determined by PSQ and MS-PCR was concordant in $85 \%$ of cases. For PSQ a cut-off of $8 \%$ was determined to best correlate with OS, using this cut-off $51 \%$ of patients were classified as methylated. For msPCR a cut-off of $13 \%$ was determined to best correlate with OS, using this cut-off $37 \%$ of patients were classified as methylated. Both methods demonstrated statistically significant correlation with OS.

\section{Author's Conclusions:}

PSQ is the ideal method due to strong interlaboratory reproducibility, increased sensitivity, and multiple studies demonstrating concordant threshold levels.

Comments and Conclusions: 
quantitative

methylation specific

PCR (msPCR)

Patient Population:

Primary GBM pts

$(\mathrm{N}=139)$

Median age at surgery:

$55.9(23-71)$
Classified as Class III, it is a multi-institutional study with a relatively large sample size, however statistical methods demonstrating superiority of one method was not performed. Note that cut-off values were determined based on FFPE and fresh frozen tissue.
Brandes, A.A et al. 2017
Study Description:

Single center retrospective study of 108 pts who underwent $\mathrm{TMZ}+\mathrm{RT}$ with recurrent GBM in whom methylation status was tested in both the primary tumor and the recurrence.

MGMT promoter methylation was detected by microscale thermophoresis

Patient Population:

Primary GBM pts

$(\mathrm{N}=178)$

Recurrent GBM pts $(\mathrm{N}=137)$

Paired GBM pts

$(\mathrm{N}=108)$

Mean age: 50.8
III

Results:

MGMT promoter was methylated in $44.4 \%$ (79) of primary GBMs and unmethylated in $55.6 \%$ (99). MGMT promoter was methylated in $46.7 \%$ (64) of recurrent GBMs and unmethylated in $53.3 \%$ (73). In paired samples the findings were as follow:

methylated $>$ methylated $=35.2 \%(n=38$, OS $=35.2 \mathrm{~m}$ ), unmethyl > unmethyl $=39.8 \%$ $(\mathrm{n}=43, \mathrm{OS}=20.1 \mathrm{~m})$, methylated $>$ unmethyl $=$ $14.8 \%(n=16, O S=27.3 \mathrm{~m})$, unmethyl > methylated $10.2 \%(n=11,0 S=23.3 \mathrm{~m})$. MGMT promoter methylation was stable in $75 \%$ of the cases, and there was significant concordance between the first and the second MGMT promoter methylation assessments $(\mathrm{K}=0.500$, $\mathrm{p}<.001)$. In multivariate analysis $M G M T$ promoter methylation at first surgery $(p<0.001)$ (but not at $2^{\text {nd }}$ surgery) was significantly correlated with survival.

\section{Author's Conclusions:}

MGMT promoter methylation status remains stable during the clinical course in the majority of GBM pts., thus re-testing this biomarker at recurrent does not provide further information. OS from diagnosis is correlated with MGMT promoter methylation status at $1^{\text {st }}$ surgery but not recurrence; a potential explanation for this may be that MGMT promoter methylation increased PFS but not post progression survival.

\section{Comments and Conclusions:}

Classified as Class III because study is retrospective review with a relatively large 
number of specimens in which hazard ratios were able to be determined.

Kim, B.S., et al.,

2017
Study Description:

Single center retrospective study of 61 pts who underwent gamma knife surgery (GKS) for local recurrent GBM pts to determine in MGMT promoter methylation was a prognostic marker of gamma knife radiosurgery

MGMT promoter methylation was detected by methylation specific PCR

Patient Population:

Recurrent GBM pts $(\mathrm{N}=61)$

Median age at GKS: 58
III

Results:

$41 \%$ (25) pts were methylated and 59\% (36) were unmethylated. MGMT promoter methylation status was the strongest predictor of OS $(p=0.03)$ on multivariate analysis, preplanning tumor volume was correlated with PFS $(p=0.005)$ on multivariate analysis regardless of methylation status. The time to progression was longer after GKS was longer in pts with MGMT promoter methylation.

\section{Author's Conclusions:}

MGMT promoter methylation conferred a profound delay in tumor progression compared to unmethylated (8.9 to 4.6 mo., $p=0.016$ ) and survival benefit (OS 14 vs 9 mo., $p=0.03$ ). MGMT promoter methylation was also an independent prognostic factor for progression and survival after GKS.

\section{Comments and Conclusions:}

Classified as Class III because it is a retrospective study with a small number of pts, they only measure methylation at initial resection, and did not have a control arm of untreated pts Does not address re-assessing methylation at recurrence.

Hsu, C. et al., Study Description:

Retrospective study comparing the prognostic power of MGMT promoter methylation assays including MSP, qMSP, $P S Q$, and IHC in 121 primary GBM pts.

Median age: 55 (40-65)

IDH-wt: N=111 (91.7\%)

IDH-mutant: $\mathrm{N}=10$

$(8.3 \%)$
III

Results:

Unmethylated pts:

IHC: $46.3 \%$ (of note histiocytes demonstrate strong staining and must be excluded); MSP PCR: $48.8 \%$ (59); qMSP: $50.4 \%$ (61), PSQ: $55.4 \%$ (67)

Methylated pts:

IHC: 53.7\%; MSP PCR: 51.2\% (62); qMSP: $49.6 \%$ (60), PSQ: 44.6\% (54)

Complete concordance was observed between methods in $73.6 \%$ of cases (89), of which $49.4 \%$ were methylated. Concordance among MSP, qMSP, and PSQ was observed in $81.8 \%$ (99) of cases. PSQ and MSP had the highest predictive power for PFS and OS, respectively and IHC had the lowest for both PFS and OS, but was not significantly different between methods. Pricing: IHC: \$35/test, MSP: \$100/test, qMSP: \$105/test, PSQ: \$200/test. 
Author's Conclusions:

MGMT status evaluated by IHC, MSP qMSP, and PSQ all showed significant correlation with PSF and $O S$ and their predictive powers were not significantly different.

\section{Comments and Conclusions:}

Classified as Class III because it is a retrospective study but has a relatively large study size but looks just at methylated vs unmethylated with no additional granularity.

Johannessen,
L.E.et al., 2018
Study Description:

Single center retrospective study of 48 pts with primary GBM in whom commercially available MGMT promoter methylation assay (PSQ therascreen, PSQ 96, MSP, MS-HRM, and qMSP) were compared.

III

\section{Results:}

Significant differences in OS in methylated vs. unmethylated MGMT promotor GBM was observed for the two PSQ kits $(p=0.011)$ and MSP ( $p=0.037)$, but not for MS-HRM and qMSP $(p=0.482$ and $p=0.113$, respectively). Median OS using MSP was 11.5 months for the group with unmethylated MGMT and 13.5 months for the group with methylated $M G M T$, whereas 2-year OS was $12 \%$ and $33.8 \%$, respectively. For PSQ, median OS was 11.6 months in the group with unmethylated MGMT promoter and 19.5 months in the group with methylated MGMT, whereas 2-year OS was $7.4 \%$ and $41.90 \%$, respectively.

\section{Author's Conclusions:}

PSQ is the best technique for prognostication.

Comments and Conclusions:

Classified as Class III because it is a retrospective study with a small number of pts and only univariate analysis was performed.

Napoleoni, L., Study Description: et al., 2019
Retrospective study of MGMT expression in 36 pts with progressive GBM as assessed by IHC.

MGMT was assessed by IHC
III

\section{Results:}

$19.4 \%$ (7) pts were negative for MGMT expression by IHC, and $80.6 \%$ (29) were positive. $33.3 \%$ (12) had expression in $>50 \%$ of cells and $66.7 \%(24)$ in $<50 \%, 19.4 \%$ (7) had expression in $>70 \%$ and $80.6 \%$ (<70\%). MGMT expression was shown to be significantly associated with disease control rate when expression was $<70 \%$ on multivariate analysis, but with PFS only on univariate analysis, and not significantly associated with OS. 
Patient Population:

Primary GBM pts

$(\mathrm{N}=36)$

Median age at $\mathrm{dx}: 61$

(24-75)
Author's Conclusions:

MGMT IHC high expression (>70\%) might be used as a "surrogate" negative predictor for response dd-TMZ treatments. However, validation is still a matter of debate.

\section{Comments and Conclusions:}

Classified as Class III as it is a retrospective study with a limited sample size and did not compare different methods of MGMT promoter methylation assessment.
Radke, J. et al., 2019
Study Description:

Retrospective study of 111 IDH-wt GBM pts in whom MGMT promoter methylation status was correlated with outcome to determine a predictive cut-off of clinical decision making.

MGMT promoter methylation was assessed by pyrosequencing, sqMSP, and dBisequ

Patient Population:

Primary GBM pts

$(\mathrm{N}=111)$

Median age at dx: 61.2 (18-85.4)
III

Results:

$49 \%$ (55) of pts were MGMT promoter unmethylated $(<10 \%)$, and $51 \%(56)$ were methylated $(\geq 10 \%)$. mPFS and OS in unmethylated GBM was $7.2 \mathrm{mo}$. and 13.4, for low level methylation (10-20\%) PFS and OS was 10.04 and 17.9 , respectively, and high level methylation (>20\%) has PFS and OS of 19.83 and 29.93. PFS was significantly different in all 3 groups but OS was only significantly different between unmethylated and high level methylated. Methylation was also determined by sqMSP and dBisequ and results were discordant as compared to pyrosequencing in $53.1 \%$ and $54.5 \%$ of cases, however in cases $>16 \%$ good consistency was seen between the 3 methods.

\section{Author's Conclusions:}

MGMT promoter methylation between $10-20 \%$ represents a transition zone in terms of PFS and OS relative to unmethylated or highly methylated patients. Of patients with low methylation PSQ results could only be validated in $51.5 \%$ of cases by another method to be clearly methylated. Recommend a 3-tier system of unmethylated (0-9\%), low level methylation (10-20\%), and high level methylation (>20\%).

\section{Comments and Conclusions:}

Classified as Class III as it is a retrospective single center study with a large relatively homogenous pt population.

response; CR, complete response; PR, partial response; PFS, progression free survival; PFS-6, 6 month progression free survival; OS, overall survival; CCNU, lomustine 


\begin{tabular}{|c|c|c|c|}
\hline Author (year): & Description of study: & $\begin{array}{l}\text { Data } \\
\text { class: }\end{array}$ & Conclusions: \\
\hline \multirow{2}{*}{$\begin{array}{l}\text { Lv, S. et al., } \\
2012\end{array}$} & Study Description: & III & \multirow[b]{2}{*}{$\begin{array}{l}\text { Results: } \\
\text { EGFR amplification } \\
\text { was detected in } 54 \% \\
\text { of GBMs, EGFRvIII } \\
\text { expression } 31.4 \% \text {, and } \\
\text { EGFRVIV expression in } \\
20 \% \text {. EGFRVIII and } \\
\text { EGFRVIV were } \\
\text { exclusively found in } \\
\text { EGFR amplified } \\
\text { GBMs. PTEN was } \\
\text { positive in } 21 \% \text { of pts. } \\
\text { Patients with an EGFR } \\
\text { amplification lacking } \\
\text { EGFRvIII had a } \\
\text { significantly superior } \\
\text { PFS and a better OS } \\
\text { following treatment } \\
\text { with cetuximab } \\
\text { [median PFS } 3.03 \text { vs. } \\
1.63 \text { months } \\
\text { (p=0.006); median OS } \\
5.57 \text { vs. } 397 \text { months } \\
\text { (p=0.12). Patients } \\
\text { with EGFRvIll positive } \\
\text { GBM had a worse } \\
\text { survival [median PFS } \\
1.63 \text { vs. } 3.03 \text { months } \\
\text { (p=0.01); median OS } \\
3.27 \text { vs. 5.57 months } \\
\text { (p=0.08)] }\end{array}$} \\
\hline & $\begin{array}{l}\text { Retrospective review of } 35 \text { recurrent GBM pts in a } \\
\text { phase II clinical trial of cetuximab (EGFR- } \\
\text { targeted mab) to determine correlation between } \\
\text { the EGFR gene amplification, EGFR variant III and } \\
E G F R \text { variant IV mutations, expression of PTEN } \\
\text { and IDH1 in response to cetuximab. }\end{array}$ & & \\
\hline
\end{tabular}

Author's Conclusions:

Pts with an EGFR amplification without EGFRvIII/vIV may show a higher sensitivity to cetuximab. Previous reports demonstrate PTEN expression (by IHC) in 60\% of primary gliomas and $17 \%$ of recurrent GBMs (comparable to the $21 \%$ found in this study).

Comments and Conclusions: 
Classified as Class III as it is a retrospective study with a limited sample size.

D'Alessandris, Q.G., et al., 2013
Study Description:

Single center prospective study of 10 pts with recurrent GBM in whom BEV and erlotinib were administered based on VEGF and EGFRvIII expression.

EGFRVIII was assessed by rt-PCR on primary tumor, VEGF was assessed by IHC on primary tumor

Patient Population:

Recurrent GBM pts $(\mathrm{N}=10)$

Median age $=53(30-77)$

VEGF overexpressed $\mathrm{N}=10$

EGFRVIII (+) $\mathrm{N}=4$
III

\section{Results:}

All the pts who received $\mathrm{BEV}+$ erlotinib achieved a radiological response (RR) (3 complete responses (CRs) and 1 partial response (PR) with RR and PFS- 6 of $100 \%$ (4/4 pts). Of the 6 pts treated with BEV alone, three had a radiological response (two CRs and one PR) with RR and PFS- 6 of $50 \%$ ( $3 / 6$ cases), 2 had progressive disease, and $1 \mathrm{pt} \mathrm{had}$ stable dx w/ intratumoral hemorrhage. The RR was $70 \%$ of cases $(7 / 10)$, with 5 CRs and 2 PRs. PFS-6 was $70 \%(7 / 10$ cases). Median PFS and OS were $8.0 \mathrm{mo}$. (range 3.0 to 31.0 months) and $9.5 \mathrm{mo}$. (range 5.0 to 31.0 mo.). BEV + erlotinib median PFS was 10.5 mo. (range 7.0-31.0 mo.) and OS was 17.0 mo. (range 8.0-31.0 mo.). BEV only median PFS was 5.5 mo. (range 3.0-9.0 mo.) and OS was and $6.75 \mathrm{mo}$. (range 5.015.0 mo.).

Author's Conclusions:

Expression of EGFRvIII is a reliable biomarker for activation of EGFR-related tyrosine kinase, which is the target of erlotinib. Conversely, anti-EGFR immunostaining, though indicative of 
EGFR overexpression, does not necessarily indicate activation of EGFR-related tyrosine kinase. RR and PFS-6 of $70 \%$ was achieved in the whole cohort, $100 \%$ in the group treated with bevacizumab and erlotinib, and $50 \%$ in the group treated with bevacizumab.

Comments and Conclusions:

Classified as Class III, while it is a prospective study there is a very limited number of pts.

Chi, A., et. al., Study Description: 2013
Multicenter, open-label, nonrandomized study of Dacomitinib (irreversible, small-molecule EGFR tyrosine kinase inhibitor) in 30 pts with firstrecurrent EGFR-amplified GBM who were antiVEGF naïve.

Serum extracellular vesicle-derived genes were assessed between 7 clinical responders and 7 rapid progressors

Patient populations:

Recurrent GBM pts $(n=30)$

Median age $=61$
Results:

EGFRvIll status was determined by rtPCR in $20(67 \%)$ all pts had GFR ECD hotspot mutations was determined by Sanger sequencing. Five $(17 \%)$ pts achieved PFS6, and thus, this arm did not meet the primary efficacy end point of 30\% PFS6. Presence of EGFRVIII was not associated with clinical benefit. There was no association between any ECD mutant cohort and clinical benefit. In addition, there was no association between clinical response and the presence of any EGFR mutation (EGFRvIll and/or ECD hotspot mutation; $\mathrm{P}=$ .2391. MGMT promoter methylation in the archival tumor specimen was not associated with clinical response. 
Dacomitinib reaches concentrations in contrast-enhancing tumor tissue well above the IC50 values for cells with sensitizing EGFR mutations. Presence of EGFR mutation did not predict a lack of benefit. Genes differentially expressed between clinical responders and rapid progressors. 32 genes that were significantly differentially expressed in serum extracellular vesiclederived in pts who remained stable $>6$ months (responders) compared with pts with disease progression $<3$ months (rapid progressors). LAMTOR2 (late endosomal/lysosomal adaptor, mitogenactivated protein kinase [MAPK], and mammalian target of rapamycin [mTOR] activator 2), which is an activator of MAPK and MTOR signaling, and CSF1, which encodes macrophage colony-stimulating factor (M-CSF), were elevated in rapid progressors.

Author's Conclusions:

Only a small subset of patients with EGFRamplified GBM derived a clinically meaningful benefit from Dacomitinib. We did not find $E G F R$ amplification, EGFRvIII or ECD mutation status to be 
associated with

clinical response.

Comments and

Conclusions:

Classified as Class III

because study is a retrospective study of

a small number of pts.

EGFR status was

determined on

archival tissue and

may not reflects the

pts current EGFR

status.

A.B., et al., 2015
Lassman,
Study Description:

Phase II study in 50 pts of dasatinib (multi-target tyrosine kinase inhibitor) as monotherapy for BEV-naïve recurrent GBM harboring overexpression/activity assessed by IHC of SRC, PDGFR, EPHA2, and/or c-KIT following TMZ+RT

\section{Patient Population:}

Recurrent GBM pts $(\mathrm{n}=50)$

Median age $=51(33-81), 54(26-75)$
III

Results:

$36 \%$ of pts had 1

molecular marker, $48 \%$

had 2 , and $16 \%$ had 3 . p-SRC was positive in $60 \%$ of pts, PDGFR in $52 \%$, EPHA2 in $86 \%$, and $\mathrm{c}-\mathrm{Kit}$ in $82 \%$.

Response was not seen in any pts, $24 \%$ showed stable $d x$, and $72 \%$ progressed.

Author's Conclusions:

Dasatanib failed to demonstrate efficacy as monotherapy for recurrent GBM.

Comments and Conclusions:

Classified as Class III as there was a limited number of pts and full statistical analysis to qualify as class II was not performed.

van den Bent, $\quad$ Study Description:

III

Retrospective study of 55 cases of matched recurrent glioblastomas treated with radiation and TMZ in which EGFR expression/mutation was assessed.

\section{$\underline{\text { Results: }}$}

EGFR amplification status remains stable in the majority (84\%) of tumors. The relative expression of EGFRvIII was often lower in recurrent tumors (53\% showed $a>20 \%$ 
EGFR amplification and EGFRvIII mutation was detected by qRT-PCR

Patient Population:

Recurrent GBM

- EGFR amp $(\mathrm{N}=55)$

- EGFRvIII $(\mathrm{N}=42)$

Median age: 51.2

decrease). Within the tumors expressing EGFRvIII at initial diagnosis, approximately $47 \%$ lose their EGFRvIII expression at tumor recurrence.

Author's Conclusions:

The relative stability of EGFR amplification indicates that molecular data obtained in the primary tumor can be used to predict the EGFR status of the recurrent tumor, but care should be taken in extrapolating EGFRvIll expression from the primary tumor.

Comments and Conclusions:

Classified as Class III as it is a retrospective study with a limited sample size.

Cioca, $A$, et al 2016
Study Description:

Single center retrospective study of 24 patients with recurrent GBM in whom EGFR expression was assessed by immunohistochemistry in the original and recurrent sample to assess for alterations in EGFR expression.

EGFR assessed by IHC

Patient Population:

Recurrent GBM pts $(\mathrm{N}=24)$

Median age at dx: 54.3 (26-78)
III

Results:

EGFR

immunopositivity was present in $96 \%$ of cases with newly diagnosed GBM; strong reactivity in 15 $(62.5 \%)$ cases, intermediate in 7 $(29.1 \%)$, and weak positivity in $1(4.1 \%)$. All the recurrent tumors expressed EGFR, with strong reactivity in 9 cases (37.5\%), moderate positivity in 10 cases (41.6\%) and weak positivity in 5 cases (20.8\%). Ten recurrent tumors (42\%) had a lower expression than their correspondent 
pair, 13 tumors (54\%)

had similar

expression, and only

one case (2\%) had

increased expression

at recurrence.

Author's Conclusions:

EGFR is

overexpressed in

glioblastoma and this

overexpression is

maintained but

decreased at

recurrence. Tumors

with strong EGFR

expression in the

primary tumor was

correlated with longer

relapse free interval

compared to cases

with low EGFR

expression (11.46 mo., $\mathrm{p}=0.017)$.

Comments and

Conclusions:

Classified as Class III because study is a retrospective study of a small number of pts with a suboptimal method of EGFR amplification detection. Treatment varied with $32 \%$ not given standard therapy.

SepulvedaSanchez, J.M., et al., 2017
Study Description:

Phase II clinical trial of 49 recurrent GBM pts with EGFR amplified +/- EGFRvIIImutation treated with Dacomitinib (pan-HER tyrosine kinase inhibitor).

Patient Population:

Recurrent GBM pts $(\mathrm{N}=49)$

Median age: 59 (39-81)
Results:

Median PFS of 2.7 months, with 4 pts progression free at 6 months and 3 pts were at 12 months. Median OS was 7.4 months. The best overall response included 1 complete response and 2 partial responses $(4.1 \%)$. Stable disease was observed in 12 patients $(24.5 \%)$ and progressive disease 
was seen in $61.2 \%$ of pts.

Author's Conclusions:

Despite the rationale to target EGFR in (recurrent) glioblastoma dacomitinib has limited efficacy as a single agent, even in EGFR amp +/- vIII mutation. The response to other EGFR inhibitors has also been disappointing.

Comments and Conclusions:

Classified as Class III as it is a prospective study with a limited sample size.

Felsberg, J., et al., 2017
Study Description:

Single center retrospective study of 106 IDH-wt gliomas to assess the prognostic significance of EGFR amplification and EGFRVIII mutation. Changes in EGFR amplification and EGFRvIII status from primary to recurrent glioblastomas were evaluated in 40 patients with EGFRamplified tumors and 33 patients with EGFRnonamplified tumors.

EGFR status was determined by IHC, PCR or both.

\section{Patient Population:}

EGFR amplified GBM pts $(\mathrm{N}=106)$

Recurrent GBM pts ( $\mathrm{N}=73)$

- EGFR amplified ( $\mathrm{N}=40)$

- Non-EGFR amplified ( $\mathrm{N}=33)$

Median age at dx: 63 (29-86)
III

Results:

$57 \%$ of EGFRamplified glioblastomas were EGFRvIII-positive. EGFRVIII positivity was not associated with different progression-free or overall survival. EGFRvIll status was unchanged at recurrence in $88 \%$ of patients with EGFRamplified primary tumors. Four patients lost and one patient gained EGFRVIII positivity at recurrence. None of 33 EGFR-nonamplified glioblastomas acquired EGFR amplification or EGFRvIll at recurrence. PCR showed slighted increased sensitivity for EGFRvIII over IHC. 
EGFRvIII and EGFR

SNVs are not

prognostic in EGFR-

amplified

glioblastoma patients.

EGFR amplification is

retained in recurrent

glioblastomas. Most

EGFRvII-positive

glioblastomas

maintain

EGFRvIII positivity at recurrence. However, EGFRvIII expression may change in a subset of patients at recurrence, thus repeated biopsy with reassessment of EGFRvIl/ status is recommended for pts with recurrent glioblastoma to receive EGFRvIIItargeting agents.

Comments and Conclusions:

Classified as Class II because study is a retrospective study of a relatively large number of patients, however, data was not suitable for NPV, PPV, sensitivity or specificity.

Hovinga, K.E. Study Description:

et al, 2019
Retrospective study of 80 GBM pts treated with BEV for recurrence in whom genomic subtype analysis was available.

EGFR amplification was determined by FISH, EGFRvIII by IHC, MGMT promoter methylation by msPCR performed on the recurrent specimen
III

Results:

Genomic subtypes: $26 \%$ classical, $36 \%$ mesenchymal, $6 \%$ neural, and 31\% proneural. The classical subtype has a higher risk of progression on multivariate analysis $(\mathrm{p}<0.001)$. EGFR was amplified in $43 \%$ of tumors and was more often present in the classical subtype $(p<0.001)$. Amplified EGFR was associated $\mathrm{w} /$ higher risk of 
progression on

multivariate analysis

$(p=0.01)$. Multifocal

change on BEV was

seen in $92 \%$ of cases.

Author's Conclusions:

Classical subtype and EGFR gene amplification are associated with significantly shorter time to progression for patients with recurrent GBM when treated with BEV.

Comments and Conclusions:

Classified as Class III because it is a retrospective study with some factors not available on all pts and with different treatment regiments. 
Patient Population:

Recurrent GBM pts $(\mathrm{N}=80)$

Mean age at treatment: 60.4 (25.1-80.33)

Lassman, A.B., et al., 2019
Study Description:

Phase I multi-center study of depatuxizumab mafodotin + TMZ of 60 patients with EGFRamplified recurrent GBM, BEV naïve pts

EGFR status was assessed by FISH, whole exome sequencing, RT-PCR and IHC on the primary resection

Patient Population:

Primary GBM pts $(n=60)$

Median age: 56 (20-79 years)
III

Results:

$50 \%$ of GBMs harbor EGFR amplification. The median duration of response was 5.6 months $(95 \% \mathrm{Cl}=1.5$, 9.7). Notable reduction in tumor size of at least $25 \%$ was observed in $22 \%$ of patients; median time to progression in this group was 3.7 months. The overall PFS was 2.1 months and the OS was 7.4 months.

Author's Conclusions:

Depatux-m + TMZ displayed an $\mathrm{AE}$ profile similar to what was described previously. Antitumor activity in this TMZrefractory population was encouraging. No association with EGFRvIII was seen.

Comments and Conclusions:

Classified as Class III because of the relatively limited number of pts and lack of a comparative arm 


\begin{tabular}{|c|c|c|c|}
\hline $\begin{array}{l}\text { Author } \\
\text { (year): }\end{array}$ & Description of study: & $\begin{array}{l}\text { Data } \\
\text { class: }\end{array}$ & Conclusions: \\
\hline \multirow{7}{*}{$\begin{array}{l}\text { Johnson, } \\
\text { B.E., et } \\
\text { al., } 2014\end{array}$} & Study Description: & \multirow[t]{7}{*}{ III } & Results: \\
\hline & \multirow[t]{2}{*}{$\begin{array}{l}\text { Single center retrospective } \\
\text { study of } 23 \text { pts with grade } \\
\text { II gliomas at initial } \\
\text { diagnosis and their } \\
\text { recurrences resected from } \\
\text { the same patients. } \\
\text { Genome sequence } \\
\text { analysis was performed } \\
\text { on paired samples to } \\
\text { interrogate alterations. }\end{array}$} & & $\begin{array}{l}54 \% \text { of mutations were detected in the primary } \\
\text { and recurrent tumor an included IDH, TP } 53, \\
\text { ATRX. } 6 \text { tumors underwent TMZ-induced } \\
\text { hypermutation and underwent malignant } \\
\text { progression to GBM. }\end{array}$ \\
\hline & & & $\begin{array}{l}\text { Recurrent tumors are often seeded by cells } \\
\text { derived from the initial tumor at a very early } \\
\text { stage of their evolution. TMZ-induced }\end{array}$ \\
\hline & \multirow{4}{*}{$\begin{array}{l}\text { Patient Population: } \\
\text { Recurrent glioma pts } \\
(\mathrm{N}=23)\end{array}$} & & mutagenesis lead to hypermutated tumors with \\
\hline & & & \\
\hline & & & Comments and Conclusions: \\
\hline & & & $\begin{array}{l}\text { Classified as Class III because it is a } \\
\text { retrospective study with a limited number of pts. } \\
\text { It should be noted that none of these pts had an } \\
\text { initial diagnosis of GBM and not all pts } \\
\text { progressed to GBM in this study. }\end{array}$ \\
\hline \multirow{5}{*}{$\begin{array}{l}\text { Meng, J., } \\
\text { et al., } \\
2014\end{array}$} & Study Description: & \multirow[t]{5}{*}{ III } & $\underline{\text { Results: }}$ \\
\hline & $\begin{array}{l}\text { Retrospective study of } 276 \\
\text { GEO cohort and } 436 \text { TCGA } \\
\text { cohort glioma pts in which } \\
\text { a } 31 \text { gene signature was } \\
\text { assessed as a biomarker } \\
\text { for estimating OS in } \\
\text { radiation-treated pts }\end{array}$ & & $\begin{array}{l}\text { Radiosensitive pts had a superior OS compared } \\
\text { with RR pts either in radiotherapy-treated subset } \\
\text { or in the patient subset that did not receive RT. } \\
\text { Nevertheless, in the multivariate Cox regression } \\
\text { analysis to assess for independent predictors of } \\
\text { the relation between the gene signature and } \\
\text { clinicopathologic features, we found that the } \\
\text { gene signature is the strongest predictor } \\
(p=0.0093) \text { in the subgroup of patients with } \\
\text { radiotherapy, whereas it does not remain } \\
\text { significant ( } p=0.202) \text { in the non RT group when } \\
\text { taking age and KPS into account. }\end{array}$ \\
\hline & \multirow[t]{3}{*}{$\begin{array}{l}\text { Median age at dx: } 50.3 \text {; } \\
56.3\end{array}$} & & Author's Conclusions: \\
\hline & & & $\begin{array}{l}\text { The radiosensitivity gene signature is mainly } \\
\text { predictive in patients treated with radiation } \\
\text { therapy. Radioresistant phenotype was enriched } \\
\text { for genes of EMT, whereas radiosensitive } \\
\text { phenotype correlated strongly with decrease of } \\
\text { genes of EMT. }\end{array}$ \\
\hline & & & Comments and Conclusions: \\
\hline
\end{tabular}


Classified as Class III as it is a retrospective study, but does have a very large cohort, however, not all pts within the GEO cohort have GBM.

Bleeker, F.E. et al., 2014
Study Description:

Single center study of 109 patient samples and 16 high grade glioma cells lines studies with between 9 and 80 pts (159 pts in total) with primary or secondary GBM. 4 patients with recurrent GBM were also included with comparison to the primary specimen.

Examined 174 exons including the following genes: IDH1, IDH2, NRAS, PTEN, TP53, AKT2, ATM, ATR, BRAF, $B R D 2, D D R 1, D Y R K 2$, EGFR, EPHA3, EPHA5, EPHA6, EPHB2, ERBB2, ERBB4, FGFR1, FGFR2, FGFR3, FGFR4, FLT1, FLT3, FRAP1, IDH1, IDH2, $K D R, K I T, M A P 2 K 4, M E T$, NRAS, NTRK2, NTRK3, PAK4, PDGFRA, PDPK1, PIK3CA, PTEN, RPS6KC1, STK11, TGFBR2 and TP53.
II

Results:

No additional mutations were observed in the four recurrent tumors compared to their primary. $67 \%$ of GBMs displayed at least one somatic mutation. Somatic mutations were found in TP53 (61 mutations), PTEN (39), IDH1 (20), PIK3CA (13), EGFR (7), BRAF(3), EPHA3 (1), NRAS (1), TGFRB2 (1), FLT3 (1) and RPS6KC1 (1). PIK $3 C A$ and PTEN mutations were mutually exclusive.

\section{Author's Conclusions:}

Most of these mutations likely represent 'driver' mutations. PIK3CA and PTEN mutations are mutually exclusive. Strong clustering of mutations in genes belonging to the PI3K-AKT pathway, however, due to the development of resistance mechanisms, kinase inhibition studies targeting the PI3K-AKT pathway for relapsing GBM have mostly failed thus far.

\section{Comments and Conclusions:}

Classified as Class III because study is retrospective with a limited clinical correlation. PI3K-AKT pathway is frequently mutated but no target therapies have shown promise. Testing for treatment purposes is not necessary at this time.

\section{Patient Population:}

GBM pts $(\mathrm{N}=109)$

Recurrent GBM $(\mathrm{N}=4)$

Mean age: 54 (15-81)

Li, R. et al., 2015
Study Description:

Single center retrospective study of whole transcriptome sequencing in primary and recurrent GBM. Paired tumors were analyzed for molecular subtype and biological progression
III

Results:

$36 \%$ of primary GBM belonged to the classical subtype vs $22 \%$ in recurrent GBM. $15 \%$ of primary GBMs were of the proneural subtype vs $23 \%$ of recurrent GBMs. The neural subtype made up $8 \%$ of primary GBM and $9 \%$ of recurrent GBM. $41 \%$ of primary and $45 \%$ of recurrent GBM were identified as the mesenchymal subtype. 
Patient Population:

Primary GBM pts $(\mathrm{N}=88)$

Recurrent GBM pts ( $\mathrm{N}=22)$

Median age at dx: 48
Author's Conclusions:

Gene set enrichment analysis revealed that chromatin fracture, repair, and remodeling genes were enriched in recurrent glioblastoma.

Comments and Conclusions:

Classified as Class III as it is a retrospective study with a very young population and conclusions are drawn that likely reflect reoperation bias more so than natural progression of the disease.
Kolodziej, M.A., et al., 2016
Study Description:

Single center retrospective study of 44 GBMs were analyzed for NDRG2 and NDRG4 expression (by PCR and IHC) and correlated with progression free survival

\section{Patient Population:}

Primary GBM pts $(n=40)$

Recurrent GBM pts $(\mathrm{N}=4)$

Median age at dx: 57.4
III

Results:

Low protein expression of NDRG2 with MGMT promoter methylation was associated with a poor PFS of $10 \mathrm{mo}$. vs $22 \mathrm{mo}$. with high NDRG2 protein expression and MGMT promoter methylation. NDRG2 and NDRG4 expression positively correlated with MGMT promoter methylation. Pts with low NDRG4 protein expression $(\leq 5.0)$ and MGMT promoter methylation had PFS of 12 (range $=6.9-17.1$ ) months compared with 17 (range=7.3-26.7) months for those with high NDRG4 protein expression (>5.0) and MGMT promoter methylation. The low NDRG4 protein expression in combination with unmethylated MGMT promoter was associated with a mean PFS of 8 months (range: $1.6-14.4$ ). vs. 5 (range $=0.2-9.8$ ) months for those with high protein NDRG4

\section{Author's Conclusions:}

Expression of NDRG2 and NDRG4 genes at the protein or mRNA level changes in response to radiochemotherapy and hypothesize that radio resistance of tumor cells overexpressing NDRG2 gene is associated with down-regulation of NDRG2 after neoadjuvant therapy. Low NDRG2 expression leads to longer PFS independent of methylation status. NDRG4 acts as a tumor suppressor gene in MGMT methylated cells and as an oncogene in unmethylated MGMT cells.

Comments and Conclusions:

Classified as Class III because it is a retrospective study with a moderate number of specimens
Wood, M.D., et al., 2016
Study Description:

III

Results: 
Retrospective study of 20 pts with $I D H$-wildtype recurrent GBM in which proteomic and genetic features are examined in pre and post treatment specimens.

Patient Population:

Recurrent GBM pts $(\mathrm{N}=20)$

Median age: 52.3 (31-73)

Immunopositivity for Ki-67 in $>20 \%$ of tumor cells was associated with shorter progressionfree and OS. Recurrent tumors showed decreased staining for CD34 suggesting lower vessel density. A subset of tumors showed increased staining for markers associated with the mesenchymal gene expression pattern, including CD44, phosphorylated STAT3, and YKL40. Recurrent tumors with the greatest increase in mesenchymal marker expression had rapid clinical progression, but no difference in overall survival after second surgery. There was poor correspondence between IHC (protein) and mRNA.

\section{Author's Conclusions:}

GBM progression is associated with a shift toward a mesenchymal phenotype in a subset of tumors and this may portend a more aggressive behavior.

Comments and Conclusions:

Classified as Class III as it is a retrospective study with a limited sample size.

Narsia, N., et al., 2017
Study Description:

Retrospective study of GEO cohort data to identify genetic alterations present in infiltrating gliomas.

\section{Patient Population:}

Primary GBM pts $(\mathrm{N}=58)$

Recurrent GBM ( $\mathrm{N}=19)$
III

Results:

3 genes were unique to recurrent GBM, overexpression of FTL and CTSL and underexpression of MT1JP. Recurrent GBM showed select alterations from primary GBM with enrichment of gap junction and hypoxia regulation genes, and WNT-beta catenin pathway genes and relative downregulation of cytokinecytokine receptor interaction, ERBB signaling, ERK1/2/MAPK signaling, PDGF, FGFR, syndecan, and VEGF pathway signaling.

\section{Author's Conclusions:}

Distinct gene expression pattern exists between grade II astrocytoma and GBM. Gene set enrichment analysis revealed a distinct expression pattern of transcriptional regulators in primary GBM. Further investigation into molecular processes showed that the genes involved in cell proliferation and invasion were shared across all subtypes of astrocytoma.

\section{Comments and Conclusions:}

Classified as Class III as it is a retrospective study with a limited sample size, the proportion $\mathrm{IDH}$ mutant recurrent GBM is not noted 
Byron,

S.A. et

al., 2018
Study Description:

Single center prospective study of 16 pts with recurrent GBM in whom genome wide tumor sequencing and molecular tumor board to determine feasibility of creating individual treatment plans within 35 calendar days.

Whole genome sequencing was performed on recurrent specimen

Patient Population:

GBM pts $(\mathrm{N}=16)$

Median age at dx: 51 (2966)

Results:

Therapeutically informative alterations were identified in 16 patients. The most common genes altered included EGFR, PTEN, CDKN2A, $N F 1, R B 1$, and TP53. Median time from surgery to molecular results and treatment recommendation was 27 calendar days, and was completed within 35 days for $81 \%$ of pts. In 1 pt molecular profiling needed to be repeated and in 2 pts RNA did not meet quality control measures. $47 \%$ (7) of pts decided to pursue the tumor board treatment recommendations, with 2 performing exceptionally well. Hyper-mutation is reported in 17\% of GBMs post TMZ exposure and is associated $\mathrm{w} /$ mutations in MMR genes.

\section{Author's Conclusions:}

Genome wide molecular test to guide treatment is feasibility and 2 patients showed prolonged TTP.

Comments and Conclusions:

Classified as Class III because while it is prospective only a small number of pts were included and endpoint was not amenable to class II statistics

Cimino, P.J. et al, 2018
Study Description:

4 cohort (TCGA, GGN, ARTE trial, and paired recurrent) retrospective study of $I D H$-wildtype glioblastoma were analyzed for gain of whole chromosome 1, gain of whole chromosome 19, and $C D K 4 / M D M 2$ Coamplification.

Results of the paired recurrent cohort was compared to the TCGA+GGN cohorts (who were assumed to be representative of the population).

Patient Population:

\section{Results:}

Pts in the recurrent cohort are biased to better functional status and survival tend to have more chromosomal alterations and specifically the following alterations: whole chromosome 1 gain, whole chromosome 19 gain, and/or mutations in TP53, with lack of CDK4 co-amplification (W3 subtype).

\section{Author's Conclusions:}

It is important to include molecular profiling, including copy number, when enrolling patients for clinical trials in order to balance arms and extrapolate relevance to the general glioblastoma population.

\section{Comments and Conclusions:}

Classified as Class III because study compares relatively large cohorts, however it is retrospective. 
GBM pts $(\mathrm{N}=620)$

Recurrent GBM $(\mathrm{N}=62)$

Neilsen, Study Description:

B.K., et

al., 2019
Retrospective study of 10 matched IDH-wildtype recurrent glioblastomas interrogated by genomic profiling, all pts were treated with standard chemo-radiation.

Patient Population:

Recurrent GBM $(\mathrm{N}=10)$

Median age at initial surgery: 60 (46-73)
III

\section{$\underline{\text { Results: }}$}

All matched tumor pairs demonstrated differences in GA between the primary and recurrence including one resected without any intervening therapy. Four genes that were commonly altered in both primary and recurrent GBM CDKN2A (86\%) and CDKN2B (86\%) deletions, EGFR activating mutation (52\%) or amplification (81\%), and TERT mutation (95\%). PI3K pathway activating mutations were also commonly seen in our cohort (67\%). EGFR alterations correlated with shorter pt. survival but statistics were not performed due to low pt. \#

\section{Author's Conclusions:}

Genetic alterations in GBM changed over time and with treatment, although some mutations are common to both the primary and recurrence. The loss of $C D K N 2 A$ inhibits both the p53 and Rb pathways, in cases that did not show CDKN2A deletion other mechanisms of p53 and Rb disruptions was present (CDKN2A mutation, $p 53$ $+R b$ mutations, and MDM2 + CDK4 amplifications). TERT promoter mutation was the most common mutation (20/21). Screening both the primary and recurrent GBM may provide information that guides therapy.

Comments and Conclusions:

Classified as Class III as it is a retrospective study with a limited sample size. 


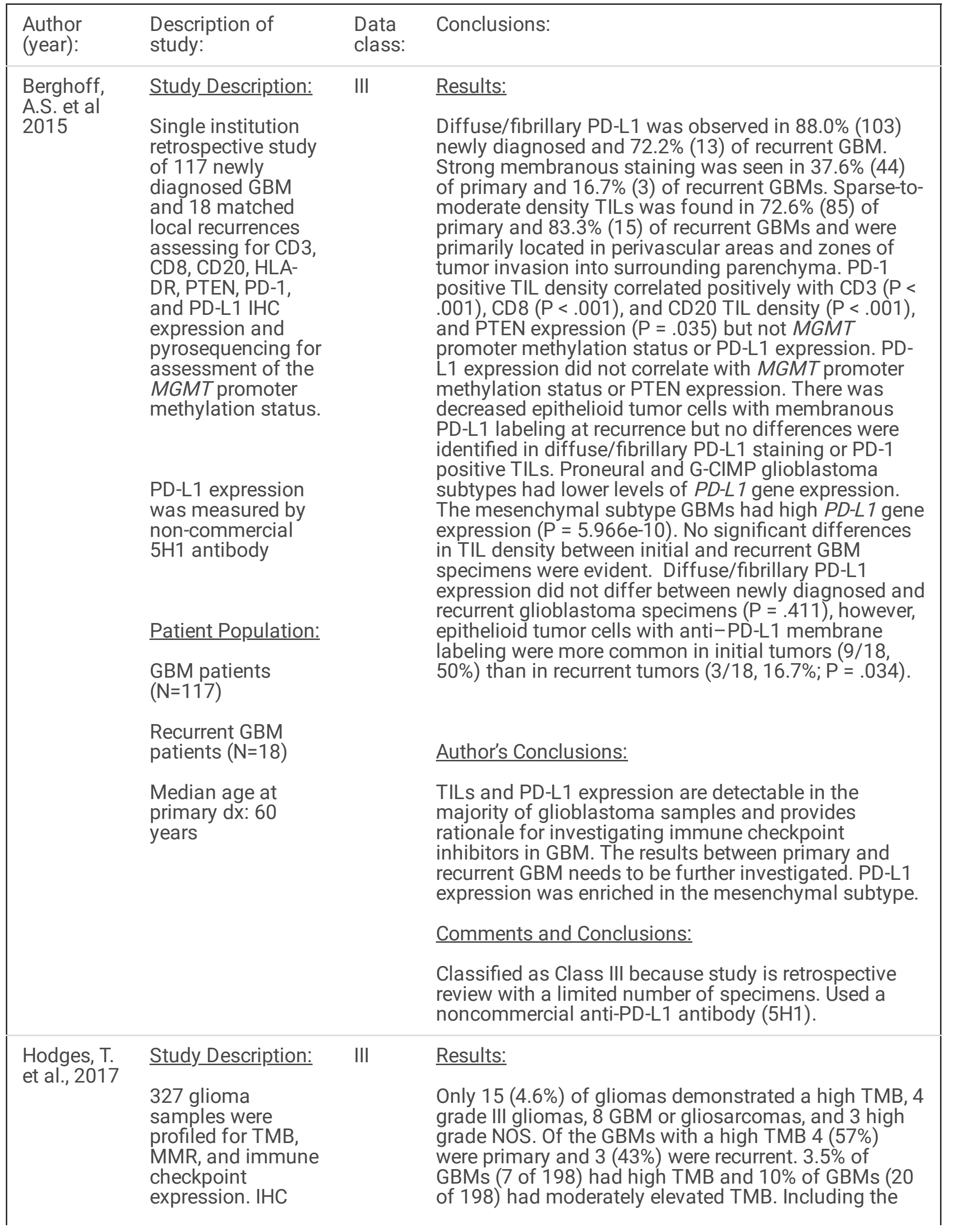


and next

generation

sequencing were

used to determine

tumor infiltrating

PD-1 positive

lymphocyte

expression, PD-L1

expression, MMR

protein expression

and mutations, and

DNA polymerase

epsilon (POLE)

mutations.

MMR IHC

performed in 30

GBMs

PD-1 staining

performed in 94

GBMs.

PD-L1 staining

performed 189

GBMs.

Patient Population:

GBM (N=198) gliosarcoma cases, $3.8 \%$ of grade IV cases (8 of 208) had high TMB and $10 \%$ (21 of 208) had moderately elevated TMB. No association between TMB and age or sex was found.

MMR protein expression was intact in $80 \%$ of GBM cases. Of the cases with loss of MMR expression $6.7 \%$ (2 of 30 ) demonstrated a loss of MLH1, 10\% a loss of MSH2 (3 of 30), 13.3\% (4 of 30) a loss of MSH6, and $6.7 \%$ (2 of 30 ) a loss of PMS2. A high TMB was significantly associated with loss of MMR IHC expression.

Of the GBM patients, 43 of 94 patients (45.7\%) were PD-1 positive and 19 of 189 patients (10.1\%) were PDL1 positive. No association was found between TMB and PD-1/PD-L1 expression.

5 of 182 (2.7\%) IDH1 wild-type GBM pts had high TMB versus 2 of $16(12.5 \%)$ IDH1 mutated GBM pts ( $P=$ .1014). PD-1 positive T cells and PD-L1 expression were predominantly detected in IDH1 wild-type tumors and were not associated with high TMB.

\section{Author's Conclusions:}

There is an association between high TMB and loss of MMR protein expression, we found no statistically significant association among $\mathrm{TMB}$, influx of cytotoxic CD8+ T cells, and immune checkpoint expression. Among the high TMB-expressing GBMs, $57 \%$ were newly diagnosed and $43 \%$ were recurrent. The recurrent GBMs with high TMB may not respond to immune checkpoint blockade, as radiation and chemotherapy may induce de novo mutations that do not elicit an effective antitumor immune response.

\section{Comments and Conclusions:}

Classified as Class III due to the limited clinical information available and particularly pertaining to this guideline the lack of investigation into alterations in progressive GBM, however there is a large sample size.

Heynckes, Study Description: III $\underline{\text { Results: }}$

S., et al., 2017

Single center retrospective study of 64 pts with primary GBM and 28 pts with recurrent GBM in which PD-L1 expression was interrogated at the protein and mRNA level.
Primary GBMs showed a highly variable expression of PD-L1 gene expression, which was decreased upon each recurrence, with a $54 \%$ decrease in median expression upon $1^{\text {st }}$ recurrence $(p=0.0041)$. PD-L1 IHC showed a similar reduction of $66.71 \%$ in recurrent tumors $(p=0.0046)$. Recurrent GBM showed a strongly reduced expression of PD-L1 (de-novo GBM $20.8 \%$, recurrent $7.6 \%$ PD-L1 positive cells) and less lymphocytic infiltration (de-novo GBM $17.5 \%$, recurrent $5.3 \%$ CD8 positive cells). Age, original surgical procedure and IDH status was not correlated with PDL1 expression. Extended TMZ therapy beyond the

Page $61 / 76$ 
PD-L1 was

measured by qRT-

PCR and E1LRN ab

by Cell Signaling

Patient Population:

Primary GBM pts

$(\mathrm{N}=64)$

Recurrent GBM pts

$(\mathrm{N}=38)$

$2^{\text {nd }}$ recurrence

$(\mathrm{N}=18)$

$3^{\text {rd }}$ recurrence

$(\mathrm{N}=10)$

Mean age at $\mathrm{dx}$ :

49
STUPP protocol was associated with PD-L1 downregulation $(p=0.02)$.

\section{Author's Conclusions:}

$20.83 \%$ of primary tumors were positive for PD-L1, with variable staining patterns. PD-L1 expression was reduced at both the mRNA and protein level in recurrent tumors. Greater reduction in PD-L1 was seen in pts with extended TMZ therapy.

\section{Comments and Conclusions:}

Classified as Class III because study is a retrospective study with a limited number of specimens.

Rahman, Study Description: III $\underline{\text { Results: }}$

M. et al.,

38 primary and 11

IDH-mutant tumors showed a lower expression of recurrent GBM cases, including 4 matched cases, were evaluated for CD3, CD8, FoxP3, CD68, CD163, PD-1, PD-L1, CTLA4 and CD70 by IHC. IDH, p53, ATRX and MGMT promoter methylation status were considered in the analysis.

Patient Population:

Primary GBM pts $(\mathrm{N}=38)$

\section{Authors Conclusions:}

Immune marker expression between primary and recurrent GBM were not significantly different. IDH-1 mutated tumors which are known to have a better prognosis were less likely to express immune checkpoint receptors, monocyte markers and 
Recurrent GBM pts $(\mathrm{N}=11)$

IDH mutant $(\mathrm{N}=9)$

Age of pts was not provided regulatory $\mathrm{T}$ cells markers which are known to be immunosuppressive.

\section{Comments and conclusions:}

Classified as class III due to the limited number of patients, particularly in the recurrent GBM group and matched samples, the lack of clinical data (including age and prior treatment). The data between matched samples is also not clearly delineated.
Lukas, R. et al, 2018
Study Description:

Phase 1a study of 16 pts with recurrent GBM previously treated with $\mathrm{RT}+\mathrm{TMZ}$ and $50 \%$ also treated with Bevacizumab who received atezolizumab (antiPD-L1) therapy.

\section{Patient Population:}

Recurrent GBM pts $(\mathrm{N}=16)$

Mean age at treatment: 52 (31-

75)

\section{Results:}

All pts had at least 1 adverse event. 16/16 (100\%) of pts discontinued therapy due to progressive disease. 1 (6\%) pt had a partial response, $3(19 \%)$ had stable disease. $3(19 \%)$ of pts had longer term survival of $16+$ months. Most pts had low or no PD-L1 expression. 12/12 tumors were microsatellite stable. $1 / 12$ patients had a hypermutated tumor, this pt showed prolonged survival (17.7 months) and decrease in the initial lesion but then demonstrated new lesions.

\section{Author's Conclusions:}

Atezolizumab is well tolerated in patients, however, there is a lack of clinical efficacy with anti-PD-1 antibody monotherapy in biomarker-unselected patients with recurrent GBM. Combined therapy or selecting for pts with MMR deficiency may be more effective.

Comments and conclusions:

Classified as class III due the limited number of pts

Omuro, A., et al., 2018

\section{Study Description: III}

Phase I clinical trial of 40 pts with recurrent GBM treated with Nivolumab monotherapy $(\mathrm{n}=10)$, Nivolumab1 + Ipilimumab3 $(n=10)$, or Nivolumab3 + ipilimumab1 $(\mathrm{N}=20)$.

\section{Results:}

$68 \%$ of patients had a PD-L1 expression level $>1 \%$ and $27 \%>10 \%$. 3 patients achieved a partial response, $1 \mathrm{pt}$ on NIVO3 $(11 \%)$ and 2 pts on NIVO3+IPI1 (10\%) and 8 had stable disease for $\geq 12$ weeks, NIVO3, $n=2(22 \%)$, NIV01+IPI3, $n=2$ (20\%), NIVO3+IPI1, n = 4 (20\%). Progressive disease was seen in $4(44 \%)$ NIVO3 pts, 7 (70\%) NIVO1+ IPI3, and 9 (45\%) NIVO3+IPI1. 68\% of pts had PD-L1 expression levels $>1 \%$ and $27 \%$ had PDL1 expression $>10 \%$ by IHC, but no association was observed between PD-L1 expression and response.

\section{Author's Conclusions:}


PD-L1 was

assessed by Dako

$\mathrm{IHC}$ on the primary

resection specimen

Patient Population:

Recurrent GBM pts

$(\mathrm{N}=40)$

Age range: $27-73$

(median 58)
Nivolumab monotherapy was better tolerated than nivolumab + ipilimumab; the tolerability of the combination was influenced by ipilimumab dose. These safety and exploratory findings merit further investigation of immunotherapies in glioblastoma. In the Phase III CheckMate 143 of Nivo3 vs BEV the endpoint of superior OS was not met

\section{Comments and Conclusions:}

Classified as Class III as it is a prospective study with a limited sample size, primarily to assess safety.
Indraccolo, Study Description: III S., et al., 2019

\section{Single center retrospective study of 57 pts having matched (diagnosis/relapse) GBM samples in which expression of MMR proteins was evaluated by $\mathrm{IHC}$, followed by whole exome sequencing.}

\section{Patient Population:}

Recurrent GBM pts $(\mathrm{N}=57)$

Mean age at treatment: 53.6
III Results:

$3.6 \%$ (2) of primary tumors had a partial loss of MMR protein pair. In recurrent GBMs 25.9\% (14) demonstrated loss of MMR IHC staining, with loss of MSH6 being the most common (12 tumors). No cases had loss of MSI. Tumors that had a complete loss of MSH6 staining by IHC showed a "hypermutant" genotype with a 135 fold increased in mutational load vs the matched primary, while tumors with only partial loss of MSH6 or no loss did not show this marked change in mutational load. The majority of cases lacking MMR protein expression at relapse (78.5\%) had methylated MGMT promoter at diagnosis. No IDH mutant tumors had loss of MMR expression $(\mathrm{N}=3)$. Telomere shortening was seen in MMR deficient tumors but not MMR intact tumors.

\section{Author's Conclusions:}

Complete loss of MSH6 correlated with increased TMB, which has been shown to correlate with response to immune checkpoint inhibitors in other tumor types. MMR deficient tumors have increased telomere shortening which might underscore genomic instability. Immune-checkpoint inhibitors have to this point not shown marked improvement over BEV, and further efforts to identify patients who would be good responders should include MMR expression, TMB, and MHC class I expression.

\section{Comments and Conclusions:}

Classified as Class III because it is a retrospective study with a limited number of pts.

\section{Results:}

Pts in the neoadjuvant arm demonstrated a statistically significant increase in OS with a hazard ratio of 0.39 compared to the adjuvant only group. Pts 
resectable GBM

pts. 19 of whom

were treated with

adjuvant post-

surgery

pembrolizumab

and 16 who were

treated with

neoadjuvant and

adjuvant post-

surgery

pembrolizumab.

Patient population:

Recurrent GBM pts

$(\mathrm{N}=35)$

Mean age at

enrollment: $\mathbf{5 7 . 4}$

IDH wild type $\mathrm{N}=$

25 (78\%)

IDH mutant $\mathrm{N}=5$ (16\%)

IDH status

unknown $\mathrm{N}=2(6 \%)$ in the adjuvant-only group had a mOS of 228 days ( 7.5 mo.), whereas those in the neoadjuvant arm had a mOS of 417 days (13.7 mo.). mPFS was 72.5 days (2.4 mo.) in the adjuvant-only group and $99.5 \mathrm{~d}$ (3.3 mo.) in the neoadjuvant group $(P=0.03)$. In pts that received surgery and had histologic evidence of tumor ( $n=15$ patients per group), the mos of the neoadjuvant treatment cohort was 400 days (13.2 mo.) from registration date, while that of the adjuvant treatment cohort was $192 \mathrm{~d}(6.3 \mathrm{mo}$.) $(P=0.03)$.

The density of tumor-infiltrating CD8+ T cells was not different between groups but demonstrated significant variability in the neoadjuvant cohort.

\section{Author's Conclusions:}

PD-1 monoclonal antibody blockade was associated with statistically significant improvements in OS and PFS when administered in the neoadjuvant setting to pts with recurrent GBM. Neoadjuvant PD-1 monoclonal antibody blockade induces functional activation of tumor-infiltrating lymphocytes, producing an interferon response within the tumor microenvironment.

\section{Comments and Conclusions:}

Classified as Class III as it is a prospective study but has a limited number of participants and control arm is not standard therapy. PD-L1 expression was not assessed. 


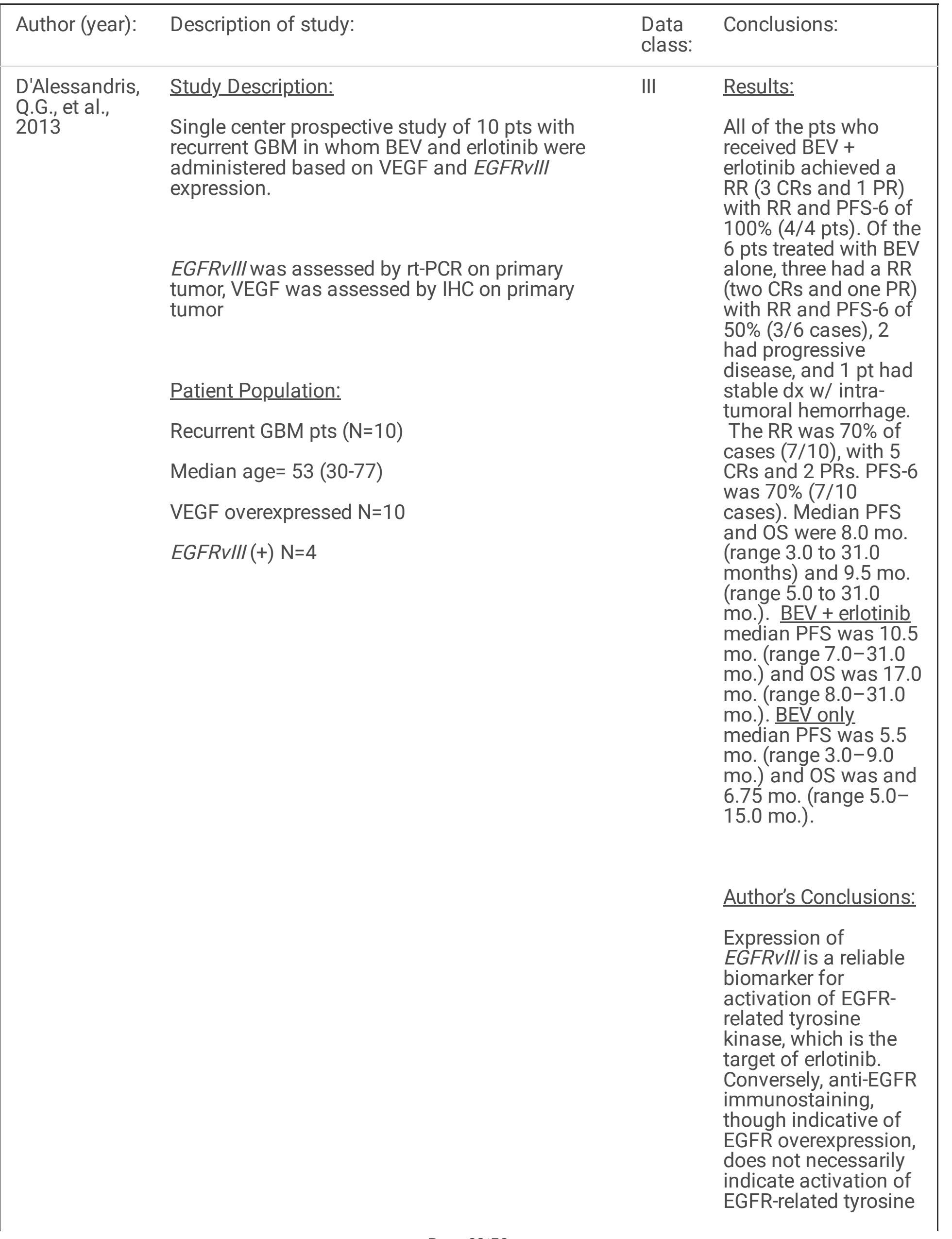


kinase. RR and PFS-6 of $70 \%$ was achieved in the whole cohort, $100 \%$ in the group treated with

bevacizumab and erlotinib, and $50 \%$ in the group treated with bevacizumab.

Comments and

Conclusions:

Classified as Class III, while it is a prospective study there is a very limited number of pts.

Takano, S, et al., 2014
Study Description:

Retrospective study of 19 pts to examine the expression of VEGF in brain tumors and if it correlates with survival.
III

III Results:

Strong expression of VEGF is seen in the blood vessels in the tumor and in the edge of GBM. In addition, strong expression is seen in the cytoplasm of tumor cells and around the areas of necrosis. Expression is weaker in the anaplastic astrocytoma and in the low-grade astrocytoma than in the GBM. VEGF is not expressed in the normal brain. VEGF concentration in the tissue of GBMs is significantly higher than that in the tissues of other types of tumors, and in the tissue of the normal brain. $\left({ }^{*} P<0.01\right)$. A VEGF concentration of more than 1,000 $\mathrm{pg} / \mathrm{mg}$ was a prognostic factor. Median OS of the patients with VEGF concentration $\geq 1,000$ $\mathrm{pg} / \mathrm{mg}$ (number [n] $=20$ ) was significantly shorter than in those with $<1,000 \mathrm{pg} / \mathrm{mg}$ 
$(\mathrm{n}=17)$ at 11.8

months and 24.8

months, respectively

$(P=0.0025)$.

Authors Conclusions:

VEGF is localized in tumor cells and tumor endothelial cells in glioma, especially in GBM, and its concentration predicts malignant glioma survival.

Comments and conclusions:

Classified as class II as it is a retrospective study with a few patients and the patient number and demographics are not clear.

Erdem-

Eraslan, L., et al., 2016
Study Description:

Retrospective study of 114 specimens from the BELOB trial. All pts were treated with temozolomide and radiotherapy and at $1^{\text {st }}$ recurrence were then treated with $\mathrm{BEV}, \mathrm{CCNU}$ or $\mathrm{BEV}+\mathrm{CCNU}$. The cohorts were interrogated to determine possible biomarkers of response to the different treatment arms.

All studies were performed on the primary tumor specimen

Patient Population:

Recurrent GBM pts $(\mathrm{N}=113)$

Median age at dx: 58 (37-77)
III

Results:

Increased

FM04/OSBPL3

expression was

significantly

correlated with

treatment response

and $O S$ in the

BEV+CCNU arm, but not the BEV or CCNU monotherapy arms.

Trend toward increased survival in pts with the "classical" subtype of GBM treated with $\mathrm{BEV}+\mathrm{CCNU}$, but it was not significant.

Author's Conclusions:

Classical GBMs showed a significant benefit in PFS and a trend toward benefit in OS from 
$\mathrm{BEV}+\mathrm{CCNU}$ treatment; other subtypes did not show such benefit. Expression of FMO4 and $O S B P L 3$, genes were associated with treatment response and increased OS in response to $\mathrm{BEV}+\mathrm{CCNU}$.

Comments and Conclusions:

Classified as Class III because study is a retrospective study of a relatively large number of $p s$.

Choi, S.W. et al., 2018
Study Description:

Single center retrospective study of 9 pts treated with $B E V$ at recurrence in whom genomic traits were analyzed to determine differences between the 2 groups. 5 pts were "BEV responders" and 4 were "non-responders".

All studies were done on the primary tumor specimen

Patient Population:

Recurrent GBM pts ( $\mathrm{N}=9$ )

Median age at dx: 58 (42-72)
III

Results:

No somatic variants were identified between the 2 groups using whole exome sequencing. 3 of 4 $(75 \%)$ of the nonresponder tumors were classified as the classical subtype, whereas only 1 of 5 (20\%) responder tumors were classical subtype. PTGS2, COL $4 A 2$, type 1 interferon pathway, immune response, and angiogenesis were significantly upregulated in the responder group, while the nonresponder group showed upregulation of angiogenesis and extracellular matrix disassembly. In an independent GBM cohort COL $4 A 2$ mRNA expression was significantly correlated with poor OS in pts who received BEV but was not prognostic when applied to pts not treated with BEV. 
Classic subtype tumors may not respond as well to $\mathrm{BEV}$. Angiogenesis related genes may be composed of distinct subgroups with distinct functional roles. COL4A2 demonstrates prognostic value in pts treated with BEV. COL $4 A 2$ encode for alpha-2 chain of type IV collagen which has been previously shown to be involved in vascular stability.

Comments and Conclusions:

Classified as Class III because study is a retrospective study of a small number of pts and analysis was performed on the primary tumor specimen

Prelaj, A., et al., 2018
Study Description:

17 recurrent GBM $(12,70.6 \%)$ and anaplastic glioma $(5,29.4 \%)$ pts, underwent first-line therapy with Stupp regimen. BEV was administered as third-line therapy after second-line therapy with FTM (13 pts) or as second-line therapy in combination with FTM (4 pts). The assessment of MGMT promoter methylation and IDH1 mutation was conducted.

Patient Population:

Recurrent GBM pts ( $\mathrm{N}=12)$

Anaplastic astrocytoma $(\mathrm{N}=5)$

Mean age at recurrence: 50 (26-66)
III

Results:

MGMT promoter was methylated in 9 patients (52.9\%) and unmethylated in 3 patients (17.5\%). The assessment of the IDH1 mutation status was conducted in 8 patients (47\%). IDH1 was mutated in 5 patients and wild-type in 3 patients.

Subgroup analysis to identify correlations between responder/nonresponder patients and clinical characteristics or tumor biomarkers such as sex, histology, tumor side, MGMT promoter methylation, IDH1 mutation and OS. A significant 
correlation between the response to $\mathrm{BEV}$ and $O S(p<0.001)$ and between the response to BEV and MGMT promoter methylation $(p<0.05)$.

Author's conclusions:

This study shows the efficacy and the safety of BEV alone or in association with FTM in the treatment of MGs.

Comments and conclusions:

Classified as Class III due to the retrospective nature with a small number of pts. More in-depth analysis of the different tumors might have been informative.

Hovinga, K.E. et al., 2019
Study Description:

Retrospective study of $80 \mathrm{GBM}$ pts treated with $B E V$ for recurrence in whom genomic subtype analysis was available.

EGFR amplification was determined by FISH, EGFRvIII by IHC, MGMT promoter methylation by msPCR performed on the recurrent specimen
III

Results:

Genomic subtypes:

$26 \%$ classical, $36 \%$ mesenchymal, $6 \%$ neural, and $31 \%$ proneural. The classical subtype has a higher risk of progression on multivariate analysis $(\mathrm{p}<0.001) . E G F R$ was amplified in $43 \%$ of tumors and was more often present in the classical subtype $(p<0.001)$. Amplified EGFR was associated $\mathrm{w} /$ higher risk of progression on multivariate analysis $(p=0.01)$. Multifocal change on BEV was seen in $92 \%$ of cases. 
Author's Conclusions:

Classical subtype and EGFR gene amplification are associated with significantly shorter time to progression for patients with recurrent GBM when treated with BEV.

Comments and Conclusions:

Classified as Class III because it is a retrospective study with some factors not available on all patients and with different treatment regiments. 
Cardona A.F et al., 2019
Study Description:

Retrospective study of 59 recurrent GBM pts treated with carmustine plus BEV as second line therapy. Response was evaluated in relation to their molecular expression profile, including CD133 mRNA, MGMT promoter methylation status, PDGFR amplification, $Y K L 40$ mRNA expression, IDH mutation, $p 53$, and EGFRvIII.

\section{Patient Population:}

Recurrent GBM pts $(n=59)$

IDH mutant pts $(\mathrm{n}=18)$

Mean age: 43 (23-70)
III

Results:

Progression-free survival of patients with gliomas derived from low-grade tumors was 14.2 months $(95 \%$ Cl $11.3-$ 17.1) and for those with primary GBMs it was 8.2 months $(95 \%$ Cl 6.2-10.1; $p=$ $0.0001)$. Almost all patients with YKL 40 overexpression exhibited higher levels of CD133 mRNA (85\%; $\mathrm{p}=0.10)$ and an absence of MGMT methylation (85.7\%; p $=0.027)$. Higher CD133 mRNA expression was correlated $(p=0.009)$ with improved PFS, while YKL4O mRNA expression correlates with a worse PFS $(p=0.01)$.

Author's Conclusions:

High YKL4O mRNA expression was related to a worse prognosis and diminished response to

BCNU/bevacizumab therapy. CD133, and YKL 40 mRNA expression have prognostic role in the response to carmustine/BEV therapy.

Comments and Conclusions:

This is classified as class III as it is retrospective and not all case may truly represent recurrent GBMs, some may 
represent anaplastic oligodendrogliomas.

Indraccolo, S., Study Description:

et al., 2019
Single center retrospective study of 57 patients having matched (diagnosis/relapse) GBM samples in which expression of MMR proteins was evaluated by IHC, followed by whole exome sequencing. IDH was assessed by sanger sequencing.

Patient Population:

Recurrent GBM pts $(\mathrm{N}=57)$

Mean age at treatment: 53.6
III

Results:

$3.6 \%$ (2) of primary tumors had a partial loss of MMR protein pair. In recurrent GBMs $25.9 \%$ (14) demonstrated loss of MMR IHC staining, with loss of MSH6 being the most common (12 tumors). No cases had loss of MSI. Tumors that had a complete loss of MSH6 staining by IHC showed a "hypermutant" genotype with a 135 fold increased in mutational load vs the matched primary, while tumors with only partial loss of MSH6 or no loss did not show this marked change in mutational load. The majority of cases lacking MMR protein expression at relapse $(78.5 \%)$ had methylated MGMT promoter at diagnosis. No IDH mutant tumors had loss of MMR expression $(\mathrm{N}=3)$. Telomere shortening was seen in MMR deficient tumors but not MMR intact tumors.

Author's Conclusions:

Complete loss of MSH6 correlated with increased TMB, which has been shown to correlate with response to immune checkpoint inhibitors in other tumor types. MMR deficient tumors have increased 
telomere shortening which might

underscore genomic instability. Immunecheckpoint inhibitors have to this point not shown marked improvement over $B E V$, and further efforts to identify patients who would be good responders should include MMR expression, $\mathrm{TMB}$, and MHC class I expression.

\section{Comments and}

Conclusions:

Classified as Class III because it is a retrospective study with a limited number of patients.

Records identified through database searching ( $n=923)$
Additional records identified through other sources

$(n=6)$

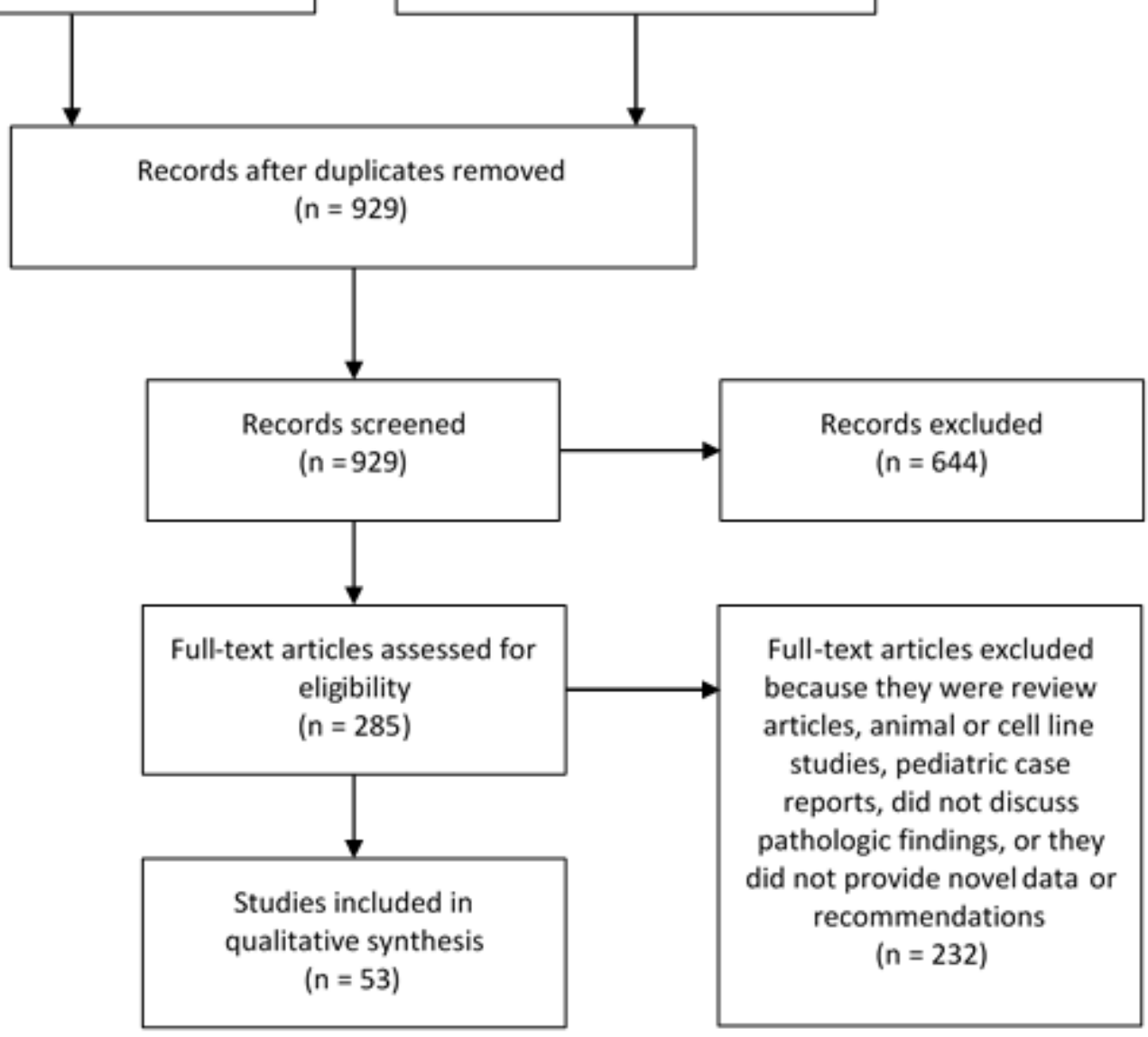


Figure 1

PRISMA Diagram: 